Preprint typeset in JHEP style - HYPER VERSION

hep-th/0407110

FREIBURG-THEP-04-06

\title{
Nut Charged Space-times and Closed Timelike Curves on the Boundary
}

\author{
Dumitru Astefanesei, ${ }^{1 *}$ Robert B. Mann ${ }^{2 \dagger}$ and Eugen Radu ${ }^{3 \ddagger}$ \\ ${ }^{1}$ Department of Physics, McGill University Montréal, Québec H3A 2T8, Canada \\ ${ }_{1,2}$ Perimeter Institute for Theoretical Physics, Ontario N2J 2W9, Canada \\ ${ }^{2}$ Department of Physics, University of Waterloo Waterloo, Ontario N2L 3G1, Canada \\ ${ }^{3}$ Department of Mathematical Physics, National University of Ireland, Maynooth, Ireland
}

\begin{abstract}
AвSTRACT: We consider higher dimensional generalizations of the four dimensional topological Taub-NUT-AdS solutions, where the angular spheres $(\theta, \varphi)$ are replaced by planes and hyperboloids. The thermodynamics of these configurations is discussed to some extent. The results we find suggest that the entropy/area relation is always violated in the presence of a NUT charge. We argue also that the conjectured AdS/CFT correspondence may teach us something about the physics in spacetimes containing closed timelike curves. To this aim, we use the observation that the boundary metric of a $(D+1)$-dimensional Taub-NUT-AdS solution provides a $D$-dimensional generalization of the known Gödel-type spacetimes.
\end{abstract}

KEYwords: AdS/CFT, Gödel Universe, zeta function regularization.

\footnotetext{
${ }^{*}$ E-mail: dastefanesei@perimeterinstitute.ca

${ }^{\dagger}$ E-mail: mann@avatar. uwaterloo.ca

${ }^{\ddagger}$ E-mail: radu@thphys.may.ie
} 


\section{Contents}

1. Introduction 1

2. A general solution 4

3. Euclidean TNAdS and thermodynamic properties 6

3.1 The four dimensional case 9

3.2 The general case 12

3.2.1 General nut solutions 13

3.2.2 General bolt solutions 14

4. Properties of the boundary metric 16

4.1 The three dimensional case

4.2 Five dimensional Gödel-type solutions 18

4.3 Properties of the general case 18

5. Discussion 20

A. Misner string analysis for general $k$

B. Scalar field in a $k=0$ Euclidean Gödel-type background 28

\section{Introduction}

The anti-de Sitter/Conformal Field Theory (AdS/CFT) correspondence [1] is the most concrete and astonishing realization of the holographic principle, which asserts that a consistent theory of quantum gravity in $(D+1)$ dimensions must have an alternate formulation in terms of a nongravitational theory in $D$ dimensions. Although string theory in AdS space is still too complicated to be dealt with in detail, in many interesting cases, it is sufficient to consider the low energy limit of the superstring theory, namely, supergravity (see ref. [2] for a review).

Using this correspondence, one can gain new insights into both theories on either side of the duality. On the one hand, quantum gravity is reformulated as an ordinary quantum field theory. This offers the possibility that a full quantum theory of gravity could be described by a CFT theory that is understood. Observables in the quantum gravity theory could be computed using quantum field theory methods. This makes it possible to attempt a microscopic analysis of the Bekenstein-Hawking entropy of asymptotically AdS black holes. On the other hand, the proposed duality has become a useful tool that can be used to learn 
something about the properties of CFTs that are only partially understood. Several gauge field theory phenomena such as confinement, confinement/deconfinement phase transitions and conformal anomalies have been shown to be encoded in the semiclassical physics of asymptotically AdS black holes [2].

The duals of AdS quantum gravity solutions are in general CFTs defined on curved backgrounds with fixed geometries. Thus, supposing the conjecture is true, we can use it to make some predictions about the basic features of a CFT in the background of a fixed boundary metric. The geometry of the boundary of an AdS solution depends on how the spacetime is sliced as one approaches the boundary, and a large variety of metrics can be obtained (see, e.g., ref. [3]). We remark that in the cases of correspondence that we do understand, the asymptotic spacetimes close to the boundary are globally hyperbolic, with a well defined notion of time. The field quantization in this case is well understood and, at least in principle, one can verify the predictions from the gravity side.

It would be interesting to understand how the AdS/CFT correspondence works in other cases, where the boundary metric presents causal pathologies, in which case usually there is no well-defined quantum field theory. An explicit example of such a situation is provided by solutions of the Einstein equations with negative $\Lambda$ and a nonvanishing NUT charge. On the supergravity side, there is nothing wrong with considering these types of configurations since the AdS/CFT correspondence permits consideration of spacetimes that asymptotically approach AdS space only locally, but not globally. In the four dimensional case, this corresponds to the AdS version of the well-known Taub-NUT solution of Einstein's vacuum equations. This metric represents a generalization of the Schwarzschild solution [4] and played an important role in conceptual developments in general relativity. For $\Lambda<0$, it has been shown [5] that this spacetime can be generalized by including an additional discrete parameter $k$. The case $k \neq 1$ corresponds to solutions where the angular spheres $(\theta, \varphi)$ are replaced by planes $(k=0)$ or hyperboloids $(k=-1)$. In the limit of vanishing NUT charge, these solutions correspond to topological black holes (see ref. [6] for reviews of the subject).

The thermodynamics of the four dimensional solutions has been discussed by several authors with some surprising features (see, e.g., refs. [3, 5]). A discussion of $(D+1)$-dimensional generalizations of the $k=1$ configurations [0] employed the Euclidean approach, continuing the time and NUT charge to imaginary values. In this case the mass parameter has to be restricted such that the fixed point set(s) of the Euclidean time symmetry is (are) regular. In four dimensions one obtains in this way two types of solutions, "nut" or "bolt", depending on whether the fixed point set has zero dimensionality (a "nut") or is of 2 dimensions (a "bolt"). In $(D+1)$ dimensions one can not only have fixed point sets of co-dimension $(D-1)$ (bolts) or zero (nuts) but also cases of intermediate dimensionality [7, 8].

The thermodynamics of the $k=1$ solutions depends on the number of spacetime dimensions. One of their more interesting features is that the entropy is not proportional to the area. The origin of this phenomenon has generally been regarded as being due to the fixed point sets of lower co-dimensionality (the nuts) which manifest themselves as Misner strings. For nut solutions in $(4 p+2)$ dimensions (with $p=1,2, \ldots)$ there are no regions in parameter 
space for which the entropy and specific heat are both positive definite, suggesting a thermodynamic instability. However, all bolt solutions have some regions of parameter space for which thermodynamic stability is possible, i.e. both entropy and specific heat are positive definite. To our knowledge, a similar discussion of the higher dimensional generalizations of the $k=0,-1$ Taub-NUT-AdS solutions is still missing in the literature.

We argue in this paper that NUT charged spacetimes are important from yet another viewpoint: namely we may hope to learn something about quantum field theories in nonglobally hyperbolic backgrounds. As noted in ref. [9] (see, also, ref. 10]), the boundary metrics of a four dimensional Lorentzian Taub-NUT-AdS spacetime correspond to the essential threedimensional part of a homogeneous Gödel-type spacetime, and contain closed timelike curves (CTCs) for a large class of parameters [11. We recall that a Gödel-type spacetime is the archetypal cosmology exhibiting properties associated with the rotation of the universe. Additionally, the Gödel model [12] is perhaps the best known example of a solution of Einstein's field equations in which causality may be violated. It thus became a paradigm for causality violation in gravitational theory. Recently there has been renewed interest in this kind of spacetime because of the discovery of a supersymmetric solution of $N=1$ five dimensional supergravity with very similar features [13]. This five dimensional solution possesses many interesting properties: it has proven to be dual to certain $p p$-waves [14, 15] and it can be extended so as to embed a black hole within it [16]. In this context, Boyda et al. [14] claimed that the chronology is well defined once a Gödel-type spacetime is prescribed with macroscopic holography: their proposal is to keep a part of the causal region associated with a comoving observer, replacing the rest with an observer dependent screen (see, also, ref. [17] for an early attempt in this direction).

Motivated by these results, we consider in this paper higher dimensional generalizations of the $k=0,-1$ topologically Taub-NUT-AdS solutions and discuss their thermodynamic properties. We find the surprising result that these solutions have an entropy that is not proportional to the area, despite the absence of Misner strings [18]. We also find that the first law of thermodynamics forces the temperature to be inversely proportional to the NUT charge; only in the $k=1$ case is the constant of proportionality forced to take on integer values so the Misner string singularities are removed. Furthermore, we find that although these solutions have non-zero angular momentum, this makes no contribution to the partition function because the angular velocity of the horizon vanishes. By studying the boundary metric of Taub-NUT-AdS spacetimes, we are led to a natural generalization in higher dimensions of the known homogeneous Gödel-type spacetimes. Not surprisingly, the line element of the five-dimensional Gödel-type supersymmetric solution found in ref. [13] is the boundary of a six-dimensional $k=0$ topological Taub-NUT-AdS solution.

The paper is structured as follows: in the next section we obtain the $(D+1)$-dimensional general solution of the Einstein equations with negative cosmological constant and nonzero NUT charge, which generalizes the known four dimensional configurations. In Section 3 we discuss its thermodynamic properties on the Euclidean section. We show that the breakdown of the entropy/area relationship does not depend upon the removal of singularities associated 
with Misner strings. As discussed in Section 4, the boundary metric of the general solution presented in Section 1 gives a higher dimensional generalization of the known Gödel-type line elements with many similar properties. In Section 5 we compile the results, proposing a preliminary discussion of the implications of the AdS/CFT correspondence for the physics in a causality violating background. Appendix A shows that Misner string singularities are absent in the $k=0,-1$ cases and appendix B contains a brief discussion of the zeta function regularization method and the Euclidean effective action for a scalar field propagating in a Gödel-type line element, explicit results being presented in a particular case.

Most of the notation and sign conventions used in this paper are similar to those in ref. [1].

\section{A general solution}

In this context, we start by considering $(D+1)$-dimensional vacuum Taub-NUT-AdS solutions, with a negative cosmological constant $\Lambda=-D(D-1) / 2 \ell^{2}$. Here $D$ is of the form $D=2 m+1$, with $m$ a positive integer. The general solution in four dimensions (which can be obtained by analytic continuation of the Euclidean solutions discussed in ref. [5]) is

$$
d s^{2}=\frac{d r^{2}}{F(r)}+\left(r^{2}+n^{2}\right)\left(d \theta^{2}+f_{k}^{2}(\theta) d \varphi^{2}\right)-F(r)\left(d t+4 n f_{k}^{2}\left(\frac{\theta}{2}\right) d \varphi\right)^{2},
$$

where

$$
F=k\left(\frac{r^{2}-n^{2}}{r^{2}+n^{2}}\right)+\frac{-2 M r+\frac{1}{\ell^{2}}\left(r^{4}+6 n^{2} r^{2}-3 n^{4}\right)}{r^{2}+n^{2}} .
$$

The discrete parameter $k$ takes the values $1,0,-1$ and implies the form of the function $f_{k}(\theta)$

$$
f_{k}(\theta)= \begin{cases}\sin \theta, & \text { for } k=1 \\ \theta, & \text { for } k=0 \\ \sinh \theta, & \text { for } k=-1\end{cases}
$$

The properties of the case $k=1$ are well known from the $\Lambda=0$ Taub-NUT solution [19, 20]. In the limit $\Lambda \rightarrow 0$, this metric has become renowned for being "a counterexample to almost anything" 21] and represents a nontrivial generalization of the Schwarzschild solution 汪. It is usually interpreted as describing a gravitational dyon with both ordinary and magnetic mass. The NUT charge $n$ plays a role dual to that of the ordinary mass $M$, in the same way that electric and magnetic charges are dual within Maxwell theory [22].

As discussed by many authors, the presence of magnetic-type mass (the NUT parameter $n$ ) introduces for $k=1$ a "Dirac-string singularity" in the metric (but no curvature singularity). This can be removed by appropriate identifications and changes in the topology of the spacetime manifold, which imply a periodic time coordinate (and thus CTCs). A similar analysis yields the same pathologies for a $k=1$ spacetime with a negative cosmological constant. However, generalizing the previous analysis for $k=1$ [20], one can prove that, for 
$k \neq 1$, there are no Misner strings even if the $(\theta, \varphi)$ section is compact (see Appendix A). Thus, there is no requisite periodicity of the time coordinate $t$, although in general there are not reasonable foliations by spacelike surfaces for any value of $k$.

In fact, it can directly be proven that, for a nonzero NUT charge, a negative cosmological constant implies the possible existence of CTCs in the bulk for a certain range of the parameters. To prove that, we consider the curve generated by the Killing vector $\partial / \partial \varphi$ and study the quantity

$$
g_{\varphi \varphi}=4 f_{k}^{2}\left(\frac{\theta}{2}\right)\left(r^{2}+n^{2}-f_{k}^{2}\left(\frac{\theta}{2}\right)\left(4 n^{2} F+k\left(r^{2}+n^{2}\right)\right)\right) .
$$

One can see that, for all $k=1,0$ metrics and those $k=-1$ metrics with $4 n^{2} / \ell^{2}>1$, the curve $r=r_{0}, t=$ const., $\theta=\theta_{0} \neq 0$ becomes timelike for large enough values of $\left(r_{0}, \theta_{0}\right)$, corresponding to a CTC since $\varphi$ is a periodic coordinate. This type of causality violation is familiar from the study of the Gödel spacetime. There are also regions of these spacetimes which are free of CTCs.

The $k=-1$ metrics with $4 n^{2} / \ell^{2} \leq 1$ are globally hyperbolic, $f\left(x^{i}\right)=t$ being a global time coordinate $\left(i . e . g^{i j}\left(\partial f / \partial x^{i}\right)\left(\partial f / \partial x^{j}\right)<0\right.$ everywhere). In the limit $n \rightarrow 0$, the $k=0,-1$ metrics correspond to topological black holes and have been considered in AdS/CFT context by many authors (see, e.g., ref. [23]).

Higher dimensional generalizations of the line element (2.1) in the $k=1, \Lambda=0$ case were first considered by Bais and Batenberg [24]. A negative cosmological constant was introduced by Awad and Chamblin [25], also only for $f_{1}(\theta)=\sin \theta$. While there are a number of papers dealing with the properties of the $k=1$ solutions with NUT charge [26], the cases $k=0,-1$ have received relatively little attention (however, see ref. [8]).

The general form for the Lorentzian Taub-NUT-AdS class of metrics for a $U(1)$ fibration over $\left(M^{2}\right)^{\otimes p}$ is

$$
d s^{2}=\frac{d r^{2}}{F(r)}+G(r)\left(d \theta_{i}^{2}+f_{k}^{2}\left(\theta_{i}\right) d \varphi_{i}^{2}\right)-F(r)\left(d t+4 n f_{k}^{2}\left(\frac{\theta_{i}}{2}\right) d \varphi_{i}\right)^{2},
$$

with $i$ summed from 1 to $p$. The base space $M^{2}$ corresponds to a two dimensional sphere $(k=1)$, plane $(k=0)$ or pseudohyperboloid $(k=-1)$.

By solving the Einstein equation in $(D+1)$ dimensions with a negative cosmological constant, we find $G(r)=r^{2}+n^{2}$, while the general form for $F(r)$ is found to be

$$
F(r)=\frac{r}{\left(r^{2}+n^{2}\right)^{(D-1) / 2}}\left(\int^{r}\left[k \frac{\left(s^{2}+n^{2}\right)^{(D-1) / 2}}{s^{2}}+\frac{D}{\ell^{2}} \frac{\left(s^{2}+n^{2}\right)^{(D+1) / 2}}{s^{2}}\right] d s-2 M\right),
$$

where the parameter $M$ is an integration constant related to the spacetime mass. These spacetimes contain only quasiregular singularities, which are the end points of incomplete and inextensible geodesics that spiral infinitely around a topologically closed spatial dimension. Moreover the world lines of observers approaching these points come to an end in a 
finite proper time [27]. The Riemann tensor and its derivatives remain finite in all parallelly propagated orthonormal frames, and no curvature scalars diverge, making them the mildest kind of singularity.

On the Lorentzian section, the parameters $M$ and $n$ are no longer related but they can be freely specified (for $k=-1, M$ may take negative values). The roots of $g_{t t}=-F(r)$ correspond to horizons, while the horizon region is always nonsingular. Again, requiring the absence of singularities due to the Misner-string implies for $k=1$ a periodicity $2 \pi(D+1) n$ of the time coordinate. For $k=0,-1$ the fibration is trivial: there are no Misner string singularities and no periodicity of $t$. However, similar to the four dimensional case, $t$ is a global time coordinate for those $k=-1$ metrics with $2(D-1) n^{2} / l^{2}<1$ only. Also, all $k=1,0$ metrics and those $k=-1$ configurations with $4 n^{2} / \ell^{2}>1$ have $g_{\varphi_{i} \varphi_{i}}<0$ for some range of $r, \theta_{i}$ (thus there are CTCs extending to infinity). In the limit $n=0$ we find the black hole solutions [6]

$$
d s^{2}=\frac{d r^{2}}{F(r)}+r^{2}\left(d \theta_{i}^{2}+f_{k}^{2}\left(\theta_{i}\right) d \varphi_{i}^{2}\right)-F(r) d t^{2}, \quad \text { with } \quad F(r)=\frac{k}{D-2}-\frac{2 M}{r^{D-2}}+\frac{r^{2}}{\ell^{2}},
$$

with $M=0$ corresponding to a special foliation of the $\operatorname{AdS}_{D+1}$ spacetime.

\section{Euclidean TNAdS and thermodynamic properties}

For solutions with NUT charge, most of the calculations in the literature are carried out for the Euclidean section. The usual approach to quantum gravity in this case is to analytically continue in the time coordinate and the NUT charge [28]

$$
t \rightarrow i \tau, \quad n \rightarrow i N
$$

in order to obtain a solution on the Euclidean section of the Einstein equations (with negative cosmological constant). Thus, the Euclidean section of the general Taub-NUT-AdS solution (2.4) reads

$$
d s^{2}=\frac{d r^{2}}{F(r)}+\left(r^{2}-N^{2}\right)\left(d \theta_{i}^{2}+f_{k}^{2}\left(\theta_{i}\right) d \varphi_{i}^{2}\right)+F(r)\left(d \tau+4 N f_{k}^{2}\left(\frac{\theta_{i}}{2}\right) d \varphi_{i}\right)^{2},
$$

where now

$$
F(r)=\frac{r}{\left(r^{2}-N^{2}\right)^{(D-1) / 2}}\left(\int^{r}\left[k \frac{\left(s^{2}-N^{2}\right)^{(D-1) / 2}}{s^{2}}+\frac{D}{\ell^{2}} \frac{\left(s^{2}-N^{2}\right)^{(D+1) / 2}}{s^{2}}\right] d s-2 M\right),(3
$$

and the radial coordinate is greater than the largest root of $F(r)$. The function $F(r)$ can be written in a more transparent way

$$
\begin{aligned}
F(r)= & \frac{r^{2}-N^{2}}{\ell^{2}}-\frac{2 M r}{\left(r^{2}-N^{2}\right)^{(D-1) / 2}}+\left(k-\frac{N^{2}(D+1)}{\ell^{2}}\right) \\
& \times \frac{r}{\left(r^{2}-N^{2}\right)^{(D-1) / 2}}\left(\int^{r} d s \frac{\left(s^{2}-N^{2}\right)^{(D-1) / 2}}{s^{2}}\right) .
\end{aligned}
$$


The properties of the resulting configurations in four dimensions have been extensively discussed by a number of authors [3, 26]. For example it was found that these Euclidean solutions cannot be exactly matched to AdS at infinity.

There is an important feature that distinguishes the solutions with $k=1$ from solutions with $k=0,-1$. The $k=1$ Euclidean solutions have the coordinate $\tau$ identified with the period

$$
\beta=\left|\frac{2 \pi N}{\sigma}\right|(D+1),
$$

which is determined by demanding regularity of the manifold so that the singularities at $\theta_{i}=0, \pi$ are coordinate artifacts ( $\sigma$ is a constant, see below). This property is not shared by $k=0,-1$ solutions since there are no Misner strings in these cases (see Appendix A). However we will find that the self-consistency of the thermodynamic relations forces eq. (3.5) to hold for these two cases as well.

For any value of $k$, the absence of conical singularities at the roots $r_{+}$of the function $F(r)$ imposes a periodicity in the Euclidean time coordinate

$$
\beta=\left|\frac{4 \pi}{F^{\prime}\left(r_{+}\right)}\right| .
$$

Usually, $\beta$ is interpreted as the inverse temperature. For $k=1$, the parameter $\sigma$ must be integer-valued so that the periodicity in $\tau$ induced by eq. (3.5) is commensurate with the periodicity induced by eq. (3.6). No such restriction on $\sigma$ holds for the $k=0,-1$ cases.

For any $k$, the mass parameter $M$ must be restricted such that the fixed point set of the Killing vector $\partial_{\tau}$ is regular at the radial position $r=r_{+}\left(\right.$defined by setting $\left.F\left(r_{+}\right)=0\right)$. We find in this way two types of solutions, "bolt" (with arbitrary $r_{+}=r_{b}>N$ ) or "nut" $\left(r_{+}=N\right)$, depending on whether the fixed point set is of dimension $(D-1)$ or is less than this maximal value.

The Lorentzian solutions (2.4) extremize the gravitational action 29]

$$
I=I_{B}+I_{\partial B}
$$

where

$$
\begin{aligned}
I_{B} & =-\frac{1}{16 \pi G} \int_{\mathcal{M}} d^{D+1} x \sqrt{-g}(R-2 \Lambda), \\
I_{\partial B} & =-\frac{1}{8 \pi G} \int_{\partial \mathcal{M}} d^{D} x \sqrt{-\gamma} \Theta .
\end{aligned}
$$

The first term in eq. (3.7) is the bulk action over the $(D+1)$-dimensional manifold $\mathcal{M}$ with metric $g$ and the second term (3.8) is the surface term necessary to ensure that the EulerLagrange variation is well-defined. Here, $\gamma$ is the induced metric of the boundary and $\Theta$ is the extrinsic curvature.

The gravitational action computed in this way (even at tree-level) contains divergences that arise from integrating over the infinite volume of spacetime. In the AdS/CFT context, 
the infrared (IR) divergences of gravity are interpreted as ultraviolet (UV) divergences of the dual CFT. A well understood way ${ }^{1}$ of computing the bulk action without introducing a background is to add local counterterms into the action [31], which remove all divergences, leading to a finite action corresponding to the partition function of the CFT. Thus we have to supplement eq. (3.7) with

$$
\begin{aligned}
I_{\text {ct }}= & \frac{1}{8 \pi G} \int d^{D} x \sqrt{-\gamma}\left\{-\frac{D-1}{\ell}-\frac{\ell \Theta(D-3)}{2(D-2)} \mathrm{R}-\frac{\ell^{3} \Theta(D-5)}{2(D-2)^{2}(D-4)}\left(\mathrm{R}_{a b} \mathrm{R}^{a b}-\frac{D}{4(D-1)} \mathrm{R}^{2}\right)\right. \\
& +\frac{\ell^{5} \Theta(D-7)}{(D-2)^{3}(D-4)(D-6)}\left(\frac{3 D+2}{4(D-1)} \mathrm{RR}^{a b} \mathrm{R}_{a b}-\frac{D(D+2)}{16(D-1)^{2}} \mathrm{R}^{3}\right. \\
& \left.\left.-2 \mathrm{R}^{a b} \mathrm{R}^{c d} \mathrm{R}_{a c b d}-\frac{D}{4(D-1)} \nabla_{a} \mathrm{R}^{a} \mathrm{R}+\nabla^{c} \mathrm{R}^{a b} \nabla_{c} \mathrm{R}_{a b}\right)+\ldots\right\},
\end{aligned}
$$

where $\mathrm{R}$ and $\mathrm{R}^{a b}$ are the curvature and the Ricci tensor associated with the induced metric $\gamma$. The series truncates for any fixed dimension, with new terms entering at every new odd value of $D$, as denoted by the step-function $(\Theta(x)=1$ provided $x \geq 0$, and vanishes otherwise).

Using these counterterms, one can construct a divergence-free boundary stress tensor from the total action $I=I_{B}+I_{\partial B}+I_{c t}$ by defining a boundary stress-tensor

$$
T_{a b}=\frac{2}{\sqrt{-h}} \frac{\delta I}{\delta h^{a b}}
$$

whose explicit expression for $D \leq 8$ is given in ref. [32]. Thus a conserved charge

$$
\mathfrak{Q}_{\xi}=\oint_{\Sigma} d^{D-1} S^{a} \xi^{b} T_{a b}
$$

can be associated with a closed surface $\Sigma$ (with normal $n^{a}$ ), provided the boundary geometry has an isometry generated by a Killing vector $\xi^{\mu}$ [33]. If $\xi=\partial / \partial t$ then $\mathfrak{Q}$ is the conserved mass/energy $\mathfrak{M}$. The angular momentum $J_{i}$ along the $i$ direction is usually associated with the Killing vector $\Psi=\partial / \partial \varphi_{i}$ - although for the metric form (2.4), a more natural choice is $\partial / \partial \varphi_{i}-2 k N \partial / \partial t$ [20].

We can proceed further by formulating gravitational thermodynamics via the Euclidean path integral

$$
Z=\int D[g] D[\Psi] e^{-I[g, \Psi]} \simeq e^{-I}
$$

where one integrates over all metrics and matter fields between some given initial and final Euclidean hypersurfaces, taking $\tau$ to have some period $\beta$, generally determined by demanding regularity of the Euclideanized manifold at degenerate points of the foliation. Semiclassically the result is given by the classical action evaluated on the equations of motion, and yields to this order an expression for the entropy

$$
S=\beta\left(\mathfrak{M}-\mu_{i} \mathfrak{C}_{i}\right)-I,
$$

\footnotetext{
${ }^{1}$ This method works, also, for some asymptotically non anti-de Sitter spaces (see, e.g., ref. [30] and references therein).
} 
upon application of the Gibbs-Duhem relation to the partition function [34] (with chemical potentials $\mathfrak{C}_{i}$ and conserved charges $\mu_{i}$ ). The specific heat $C$ is computed with the standard thermodynamic formula

$$
C=-\beta \frac{\partial S}{\partial \beta}
$$

In ref. [7], the action, mass/energy, entropy and specific heat of the spherically $(D+1)$-dimensional Taub-NUT-AdS solutions were calculated in this way.

\subsection{The four dimensional case}

We start by applying this procedure to the better-known four dimensional case.

The $k=1$ solutions have an intrinsic Euclidean time periodicity $\beta=8 \pi N$, fixed by demanding the absence of Dirac string singularities. For $k=0,-1$ the fibration is trivial and the periodicity of $\beta$ is not fixed by this requirement. Detailed analyses of the $k=1$ nut and bolt solutions were presented in refs. 3, 5, 7] and we shall not repeat them here.

The mass/energy of the topological TNAdS solutions calculated by the counterterm formalism is $\mathfrak{M}=V M /(4 \pi G)$, which yields the familiar $D=3$ expression for the topological black hole mass in the limit $N \rightarrow 0$ (in this section, $V$ is the dimensionless area of the $\theta, \varphi$ space, and can be normalized to $4 \pi$ ).

Interestingly enough, since asymptotically

$$
T_{\varphi t}=\frac{8 M n f_{k}^{2}(\theta / 2)}{\ell r}+O\left(1 / r^{2}\right), \quad T_{t t}=\frac{2 M}{\ell r}+O\left(1 / r^{2}\right),
$$

these solutions also have a nonzero angular momentum density, while the total angular momentum is obtained by computing eq. (3.11). This quantity vanishes for the $k=1$ solution, but yields a nonzero value $J \sim n M$ in the other two cases.

To compute the associated angular velocity $\Omega$ entering the thermodynamics, we consider a family of locally nonrotating observers "at rest" with respect to the $t=$ const. hypersurface [35], with velocity $u^{a} \sim \nabla^{a} t$. These observers rotate with an angular velocity $\Omega=-g_{t \varphi} / g_{\varphi \varphi}$. Unlike the Kerr-AdS case, this vanishes as $r \rightarrow r_{+}$. Thus the contribution of $J$ to the thermodynamic partition function is zero. ${ }^{2}$

Nut solutions have fixed point sets at $r=N$. The mass parameter of planar nut solutions $(k=0)$ must take the value $M_{n}=-4 N^{3} / \ell^{2}$, while the metric function $F(r)$ takes the form

$$
F(r)=\frac{(r-N)^{2}(r+3 N)}{\ell^{2}(r+N)}
$$

with a double zero at $r=N$. As a result, these solutions correspond to a background with zero temperature [5], since the Euclidean time $\tau$ can be identified with any period $\beta$. The action and the entropy of this solution are

$$
I_{0,4}^{N}=-\frac{V \beta}{4 \pi G} \frac{N^{3}}{\ell^{2}}, \quad S_{0,4}^{N}=-\frac{3 V \beta}{4 \pi G} \frac{N^{3}}{\ell^{2}} .
$$

\footnotetext{
${ }^{2} \mathrm{~A}$ similar situation has been found for some asymptotically flat rotating black holes in an Einstein-Maxwelldilaton theory [36].
} 
It can be verified that the first law of thermodynamics $d S=\beta d \mathfrak{M}$ is satisfied, since we can always take $\beta=\sigma N$, with $\sigma$ an arbitrary constant.

The situation with $k=0$ bolt solutions is different. Here, $\partial_{\tau}$ has a two-dimensional fixed point set at some radius $r=r_{b}>N$. In this case the mass parameter is

$$
M_{0,4}^{b}=\frac{1}{2 \ell^{2} r_{b}}\left(r_{b}^{4}-6 N^{2} r_{b}^{2}-3 N^{4}\right)
$$

the period $\beta$ resulting from eq. (3.6) is

$$
\beta=\frac{4 \pi \ell^{2}}{3} \frac{r_{b}}{r_{b}^{2}-N^{2}} .
$$

while the action and entropy are

$$
\begin{aligned}
I_{0,4}^{b} & =-\frac{V\left(r_{b}^{4}+3 N^{4}\right)}{12 G\left(r_{b}^{2}-N^{2}\right)}, \\
S_{0,4}^{b} & =\frac{V\left(r_{b}^{4}-4 N^{2} r_{b}^{2}-N^{4}\right)}{4 G\left(r_{b}^{2}-N^{2}\right)} .
\end{aligned}
$$

Note that, for any value of $r_{b}$, the entropy is not proportional to the area $A=V\left(r_{b}^{2}-N^{2}\right)$ of the bolt. This is quite distinct from the result obtained in computing the entropy of the planar $k=0$ bolt metric relative to its nut counterpart - upon appropriate matching of the geometries at large radius, one obtains a value of $A / 4$ for the relative entropy [5]. However, we see that the counterterm approach yields an entropy that is not proportional to the area despite the absence of Misner strings.

The situation in the hyperbolic case is somewhat special. In this case there are no nuts [5] since $F(r)$ becomes negative for values of $r$ close to (but larger than) $N$. We find only bolts at some value of $r=r_{b}>N$. The corresponding mass parameter is

$$
M_{-1,4}^{b}=\frac{1}{2 \ell^{2} r_{b}}\left(r_{b}^{4}-\left(6 N^{2}+\ell^{2}\right) r_{b}^{2}-N^{2}\left(3 N^{2}+\ell^{2}\right)\right)
$$

while the inverse temperature is given by

$$
\beta=\frac{4 \pi \ell^{2} r_{b}}{3\left(r_{b}^{2}-N^{2}\right)-\ell^{2}},
$$

which is a positive quantity, as follows from the assumptions we made on the function $F(r)$. For this value of $\beta$, the expressions of the action and entropy are

$$
\begin{aligned}
I_{-1,4}^{b} & =-\frac{V\left(r_{b}^{4}+\ell^{2} r_{b}^{2}+N^{2}\left(\ell^{2}+3 N^{2}\right)\right)}{4 G\left(3 r_{b}^{2}-\ell^{2}-3 N^{2}\right)} \\
S_{-1,4}^{b} & =\frac{V\left(\ell^{2} N^{2}+3 N^{4}+\ell^{2} r_{b}^{2}+12 N^{2} r_{b}^{2}-3 r_{b}^{4}\right)}{4 G\left(\ell^{2}+3 N^{2}-3 r_{b}^{2}\right)} .
\end{aligned}
$$



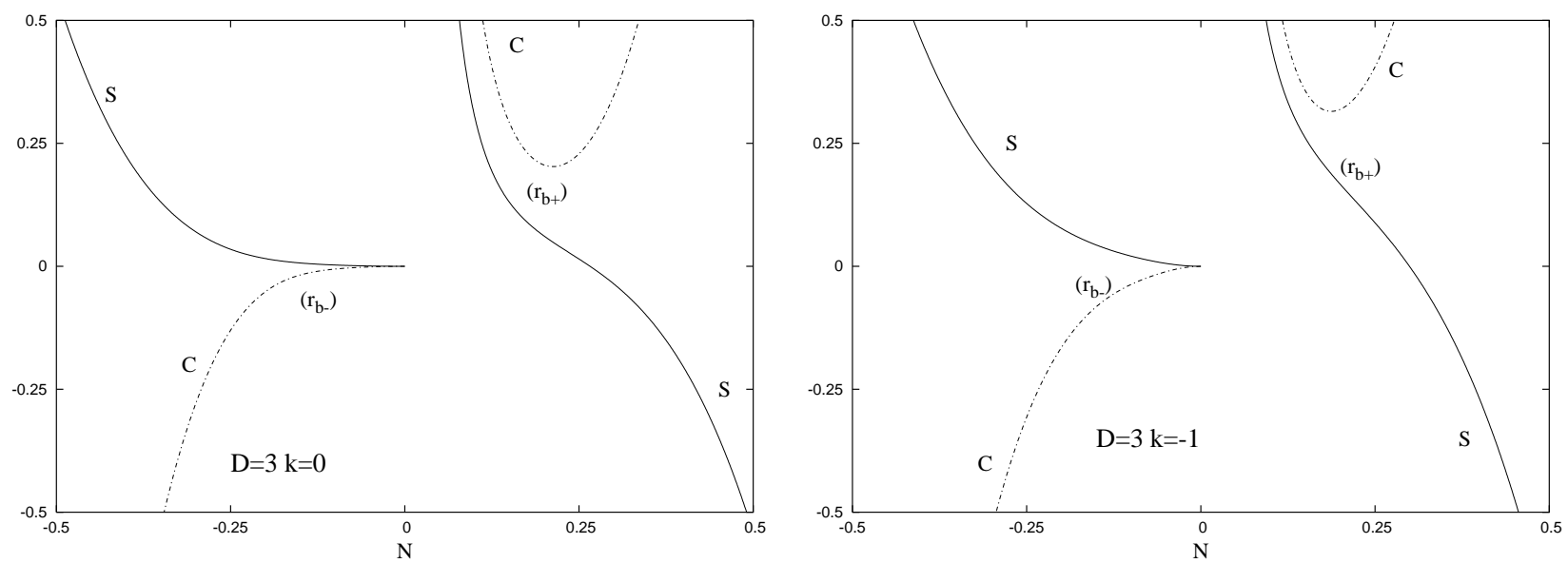

Figure 1. The entropy and specific heat of $k=0,-1$ solutions are represented as a function of $N$ for bolt solutions in four dimensions.

We can see that, given the arbitrariness in the choice of $r_{b}$, these quantities may take any values. However, for any $k$, the bolt solutions with large enough values of $r_{b}$ have $S_{k, 4}>0$ and are thermally stable $C_{k, 4}^{b}>0$ (although the action is negative).

For generic $r_{b}>N$, we find that the first law of thermodynamics is not satisfied for any value of $k$. This suggests that the bolt radius is not independent of the NUT charge and/or the cosmological constant. Supposing $r_{b}=r_{b}(N)$ we find that the first law of thermodynamics holds if and only if

$$
r_{b \pm}=\frac{\left.\sigma \ell^{2} \pm \sqrt{\sigma^{2} \ell^{4}+48 N^{2}\left(3 N^{2}-k \ell^{2}\right.}\right)}{12 N}
$$

where $\sigma$ is an arbitrary parameter. Insertion of this relationship into eq. (3.6) implies $\beta=2 \pi(D+1) N / \sigma$. Remarkably, we find that a consistent thermodynamics forces a relation between the NUT charge and the bolt radius, despite the absence of Misner string singularities in the $k=0,-1$ cases. For the $k=1$ spacetimes, these can be removed by requiring $\sigma$ to be an integer. Insertion of eq. 3.16) into the expression for the entropy yields a value that is not proportional to the area of the bolt unless $N=0$.

There are two branches that yield consistent thermodynamics. The obvious condition $r_{b}^{2}>N^{2}$ implies that the lower branch $r_{b-}$ exists for a negative $N$ only and arbitrary $\sigma$. For $N>0$, the $r_{b+}$ branch exists when $\sigma>2 k$, whereas for a negative $N$, it exists for $\sigma<2 k$.

The thermodynamic quantities of these solutions are found by replacing eq. (3.16) in the general relations (3.15), (3.16). For example, the expression of the specific heat is

$$
C=\frac{V \ell^{4}}{144 G N^{2} \sigma^{2}} \pm \frac{V\left(\ell^{8}-24 \ell^{4} N^{2} \sigma^{2}\left(k \ell^{2}-6 N^{2}\right)+10368 N^{6} \sigma^{4}\left(-k \ell^{2}+4 N^{2}\right)\right)}{144 G \ell^{2} N^{2} \sigma^{2} \sqrt{\ell^{4}+48 N^{2} \sigma^{2}\left(-k \ell^{2}+3 N^{2}\right)}} .
$$

However, the arbitrariness of $\sigma$ makes their analysis somewhat difficult and the analytical expressions are not very illuminating.

Figure 1 shows a plot of the entropy and specific heat per unit volume vs. $N$ (for $\ell=1)$. We see that for small $N$, the $k=0,-1$ upper branch solutions $\left(r_{b}=r_{b+}\right)$ are 
thermally stable, whereas the lower branch solutions $\left(r_{b}=r_{b-}\right)$ are always unstable. The upper branch quantities diverge in the high temperature limit (i.e., $N \rightarrow 0$ ) while the lower branch quantities are all zero. These results are obtained for $|\sigma|=1.5$. Similar results have been obtained for other values of $\sigma$.

\subsection{The general case}

Now we turn to the general case. For $k=1$, an expression for the mass, temperature and finite gravitational action in arbitrary dimensions was derived in ref. [7].

Not unexpectedly, the general methods presented there to find the finite values of the action and mass are generally valid for any value of the discrete parameter $k$. Therefore, we repeat here only the basic arguments put forward in Section 7 of ref. [0].

In the general case, the bulk contribution to the total action is

$$
I_{B}=\frac{D V \beta}{8 \pi G \ell^{2}} \int_{r_{+}}^{r^{\prime}} d r\left(r^{2}-N^{2}\right)^{(D-1) / 2}
$$

where $V=\int \prod_{i=1}^{p} f_{k}\left(\theta_{i}\right) d \theta_{i} d \varphi$ is the total area of the sector $\left(\theta_{i}, \varphi_{i}\right)$ (with $V=(4 \pi)^{(D-1) / 2}$ for $k=1) .{ }^{3}$ The integrand here can be expanded using the binomial theorem. Integrating term by term and taking the limit $r^{\prime} \rightarrow \infty$, we find that the general expression for the finite contribution from the bulk action is

$$
I_{B \text { finite }}=-\frac{D V \beta}{8 \pi G \ell^{2}} \sum_{i=0}^{(D-1) / 2}\left(\begin{array}{c}
\frac{(D-1)}{2} \\
i
\end{array}\right)(-1)^{i} N^{2 i}\left[\frac{r_{+}^{D-2 i}}{D-2 i}\right],
$$

with $r_{+}$the value of $r$ that is the largest positive root of $F(r)$. The expression for the trace of the extrinsic curvature $\Theta$ corresponding to the metric (3.2) is

$$
\Theta=\frac{F^{\prime}(r)}{2 \sqrt{F(r)}}+(D-1) \frac{r \sqrt{F(r)}}{\left(r^{2}-N^{2}\right)} .
$$

Thus, expanding $\sqrt{-\gamma} \Theta$ for large $r$, the general finite contribution from the boundary action is

$$
I_{\partial B \text { finite }}=\frac{D V \beta}{8 \pi} M
$$

To evaluate $I_{c t}$ we make use of the expression of the Ricci scalar of the $D$-dimensional boundary metric

$$
\mathrm{R}=(D-1)\left(\frac{k}{\left(r^{2}-N^{2}\right)}-\frac{F(r) N^{2}}{\left(r^{2}-N^{2}\right)^{2}}\right)
$$

Using that relation and the expressions for the Ricci tensor of the boundary metric, we find that for every $k$, the only finite contribution from the counterterm action 3.10) comes from

\footnotetext{
${ }^{3}$ In the non-compact case, $V$ will diverge. If we compactify, then it will be finite. However, in the divergent case, we can consider entropy and action per unit $V$.
} 
the first term as $r \rightarrow \infty$. All of the other terms will either go to zero or diverge, and will be used to cancel the divergences in $I_{B}$ and $I_{\partial B}$.

Thus, the general expression for the finite gravitational action in $(D+1)$ dimensions is similar to that found in ref. [0]

$$
I_{k, D+1}=\frac{V \beta}{8 \pi G}\left[M-\frac{D}{l^{2}} \sum_{i=0}^{(D-1) / 2}\left(\begin{array}{c}
\frac{(D-1)}{2} \\
i
\end{array}\right)(-1)^{i} N^{2 i} \frac{r_{+}^{(D-2 i)}}{(D-2 i)}\right] .
$$

We can also derive a general expression for the mass for the TNAdS solutions, by using the general relation (3.11). The divergence cancellations take place in a similar manner and we find for the conserved mass-energy

$$
\mathfrak{M}=\frac{(D-1) V M}{8 \pi G} .
$$

We have checked the validity of the results 3.19$)$ and $(3.20)$ for $D=3,5,7$.

However, the value of $M$ is fixed by the condition $F\left(r=r_{+}\right)=0$ to be

$$
\begin{aligned}
M_{k, D+1} & =\frac{1}{2} \int^{r_{+}} \frac{d s}{s^{2}}\left(\left(k\left(s^{2}-N^{2}\right)^{\frac{D-1}{2}}+\frac{D}{\ell^{2}}\left(s^{2}-N^{2}\right)^{\frac{(D+1)}{2}}\right)\right. \\
& =\frac{1}{2}\left(\frac{\left(r_{+}^{2}-N^{2}\right)^{(D+1) / 2}}{l^{2} r_{+}}+\left(k-\frac{N^{2}(D+1)}{l^{2}}\right) \int^{r_{+}} d s \frac{\left(s^{2}-N^{2}\right)^{(D+1) / 2}}{s^{2}}\right) .
\end{aligned}
$$

To derive the periodicity $\beta$ of the Euclidean time coordinate, we use the relations

$$
\left|\frac{d F(r)}{d r}\right|_{r=N}=\frac{2 k}{N(D+1)}
$$

while for bolt solutions

$$
\left|\frac{d F(r)}{d r}\right|_{r=r_{b}}=\frac{1}{r_{b}}\left(k+\frac{D}{\ell^{2}}\left(r_{b}^{2}-N^{2}\right)\right) .
$$

The thermodynamics of the general solution can also be discussed to some extent. For $k \neq 1$, the behavior known from four dimensions is found to be generic: there are no hyperbolic nuts with $\Lambda<0$, the $k=0$ nuts have zero temperature and the first law of thermodynamics implies that $r_{b}$ is a function of $\ell$ and $N$.

Furthermore, the $(D+1)$ dimensional solutions also have $p$ nonvanishing charges $J_{i}$, associated with the Killing vectors $\varphi_{i}$ that are interpreted as angular momenta. However, as with the $D=3$ case, the angular velocities $\Omega_{i}$ entering the thermodynamic relations are zero and so the entropy is still given by $S=\beta \mathfrak{M}-I$.

\subsubsection{General nut solutions}

We start by discussing the simpler case when the largest zero of the function $F(r)$ is at $r_{+}=N$. Since the properties of the $k=1$ solutions are discussed in ref. [0], we focus here 
on the cases $k=0,-1$. Here we remark that, as implied by eq. (3.22), $d F /\left.d r\right|_{r=N}=0$ for $k=0$ and any $D$, implying that $F(r)$ has a double zero as $r_{+}=N$. Thus, the $k=0$ NUT solutions correspond to a background of arbitrary temperature since the Euclidean time $\tau$ can be identified with arbitrary period. By using the same relation, we can prove that the "no hyperbolic nuts" result found in ref. [5] for $D=3$ also generalizes to higher dimensions with negative cosmological constant. Eq. (3.22) implies that the function $F(r)$ necessarily has a second zero for some $r_{+}>N$, and $F(r)$ is positive for $r>r_{+}$.

Making use of the relation

$$
\left.\int d x \frac{\left(x^{2}-1\right)^{n}}{x^{2}}\right|_{x=1}=-\frac{1}{\sqrt{\pi}} \Gamma\left(\frac{1}{2}-n\right) \Gamma(n+1),
$$

we can write simple expressions for the parameter $M$, action and entropy of $k=0,1$ NUT solutions

$$
\begin{aligned}
M_{k, D+1}^{N} & =\frac{\sqrt{\pi}}{2 \ell^{2}}(-1)^{(D-1) / 2} N^{D-2}\left(N^{2}(D+1)-k l^{2}\right) \frac{\Gamma\left(\frac{D+1}{2}\right)}{\Gamma\left(\frac{D}{2}\right)} \\
I_{k, D+1}^{N} & =\frac{V \beta(-1)^{(D-1) / 2}}{16 \sqrt{\pi} G \ell^{2}} N^{D-2}\left((D-1) N^{2}-k \ell^{2}\right) \frac{\Gamma\left(\frac{D+1}{2}\right)}{\Gamma\left(\frac{D}{2}\right)} \\
S_{k, D+1}^{N} & =\frac{V \beta(-1)^{(D-1) / 2}}{16 \sqrt{\pi} G l^{2}} N^{D-2}\left(D(D-1) N^{2}-(D-2) k \ell^{2}\right) \frac{\Gamma\left(\frac{D+1}{2}\right)}{\Gamma\left(\frac{D}{2}\right)}
\end{aligned}
$$

where $\beta=2 \pi N(D+1)$ for $k=1$ and is arbitrary for $k=0$. For $k=1$, we recover the relations presented in ref. [7]. In this case, for any $D$, there is no region in parameter space for which the entropy and specific heat are both positive definite. Again, for $k=0$, the first law of thermodynamics is satisfied by taking the periodicity of the Euclidean time coordinate to be $\beta=\sigma N$, with $\sigma$ an arbitrary constant.

\subsubsection{General bolt solutions}

Bolt solutions exist for every value of $k$. In this case, the condition $F\left(r_{b}>N\right)=0$ implies that

$$
\beta=\frac{4 \pi r_{b} \ell^{2}}{k \ell^{2}+D\left(r_{b}^{2}-N^{2}\right)}
$$

is the periodicity of $\tau$ coordinate. The parameter $M_{k, D+1}^{b}$ is fixed by eq. (3.21), with $r_{+}=r_{b}$. Note the existence for $k=1$ of an intrinsic periodicity $\beta=\left|\frac{2 \pi(D+1) N}{p}\right|$, which fixes the value of $r_{b}$ as a function of $(\ell, N)$.

The action and entropy of the bolt solutions are given by the general expressions

$$
\begin{aligned}
I_{k, D+1}^{b} & =\frac{V \beta}{16 \pi G \ell^{2}}\left(-\frac{\left(r_{b}^{2}-N^{2}\right)^{(D+1) / 2}}{r_{b}}+\left(k \ell^{2}-(D-1) N^{2}\right) \int^{r_{b}} d s \frac{\left(s^{2}-N^{2}\right)^{(D-1) / 2}}{s^{2}}\right), \\
S_{k, D+1}^{b} & =\frac{V \beta}{16 \pi G \ell^{2}}\left(\frac{D\left(r_{b}^{2}-N^{2}\right)^{(D+1) / 2}}{r_{b}}+\left((D-2) k \ell^{2}-D(D-1) N^{2}\right) \int^{r_{b}} d s \frac{\left(s^{2}-N^{2}\right)^{(D-1) / 2}}{s^{2}}\right) .
\end{aligned}
$$


We omit here the expression of the specific heat since it is extremely complicated and not particularly enlightening.

As found in ref. [7], all $k=1$ bolt solutions have some regions of parameter space for which thermodynamic stability is possible. As expected, this property is shared by all $k=0,-1$ counterparts. The existence in this case of three essential parameters $\left(r_{b}, N, \ell\right)$ makes this fact always possible.

We present the expression of the relevant quantities in six dimensions

$$
\begin{aligned}
M_{0,6}^{b} & =\frac{1}{2 \ell^{2} r_{b}}\left(r_{b}^{6}-5 N^{2} r_{b}^{4}+15 N^{4} r_{b}^{2}+5 N^{6}\right), \\
I_{0,6}^{b} & =\frac{V\left(-3 r_{b}^{6}+5 N^{2} r_{b}^{4}+15 N^{4} r_{b}^{2}+15 N^{6}\right)}{60 G\left(r_{b}^{2}-N^{2}\right)} \\
S_{0,6}^{b} & =\frac{V\left(3 r_{b}^{6}-13 N^{2} r_{b}^{4}+33 N^{4} r_{b}^{2}+9 N^{6}\right)}{12 G\left(r_{b}^{2}-N^{2}\right)}
\end{aligned}
$$

for planar bolts, and

$$
\begin{aligned}
M_{-1,6}^{b} & =\frac{1}{6 \ell^{2} r_{b}}\left(3 r_{b}^{6}-\left(15 N^{2}+\ell^{2}\right) r_{b}^{4}+\left(45 N^{4}+6 \ell^{2} N^{2}\right) r_{b}^{2}+15 N^{6}+3 \ell^{2} N^{4}\right) \\
I_{-1,6}^{b} & =\frac{V\left(-3 r_{b}^{6}+\left(5 N^{2}-l^{2}\right) r_{b}^{4}+3 N^{2}\left(5 N^{2}+2 \ell^{2}\right) r_{b}^{2}+3 N^{4}\left(5 N^{2}+\ell^{2}\right)\right)}{12 G\left(r_{b}^{2}-N^{2}\right)} \\
S_{-1,6}^{b} & =\frac{V\left(15 r_{b}^{6}-\left(65 N^{2}+3 \ell^{2}\right) r_{b}^{4}+3 N^{2}\left(55 N^{2}+6 \ell^{2}\right) r_{b}^{2}+9 N^{4}\left(5 N^{2}+\ell^{2}\right)\right)}{12 G\left(r_{b}^{2}-N^{2}\right)}
\end{aligned}
$$

for the six-dimensional hyperbolic bolts. One can see that, for large enough values of $r_{b}$ the action is negative, while the mass parameter and entropy become positive.

However, as with the four dimensional case, the first law of thermodynamics is satisfied provided $r_{b}$ is a function of $N$, which in turn implies that eq. (3.5) holds.

We find the existence of two branches, given by

$$
r_{b \pm}=\frac{\sigma \ell^{2} \pm \sqrt{\sigma^{2} \ell^{4}+D(D+1)^{2} N^{2}\left(D N^{2}-k \ell^{2}\right)}}{D(D+1) N} .
$$

Unlike from the case $k=1$, the parameters $N, \ell$ may take arbitrary values. Several restrictions appear from the condition $r_{b}>N$. For the upper branch $r_{b+}$, a positive $N$ implies values of $\sigma>k(D+1) / 2$, while, for a negative $N$, we find the condition $\sigma<k(D+1) / 2$.

The lower branch exists for an arbitrary $\sigma$ but negative values of $N$ only.

We have looked for the thermodynamic quantities in a number of dimensions up to $D=9$ and found a general picture that resembles the $D=3$ case. For small $N$, the $k=0,-1$ upper branch solutions are thermally stable, whereas the lower branch solutions are always unstable. The upper branch quantities diverge in the high temperature limit while the lower branch quantities vanish in the same limit. 

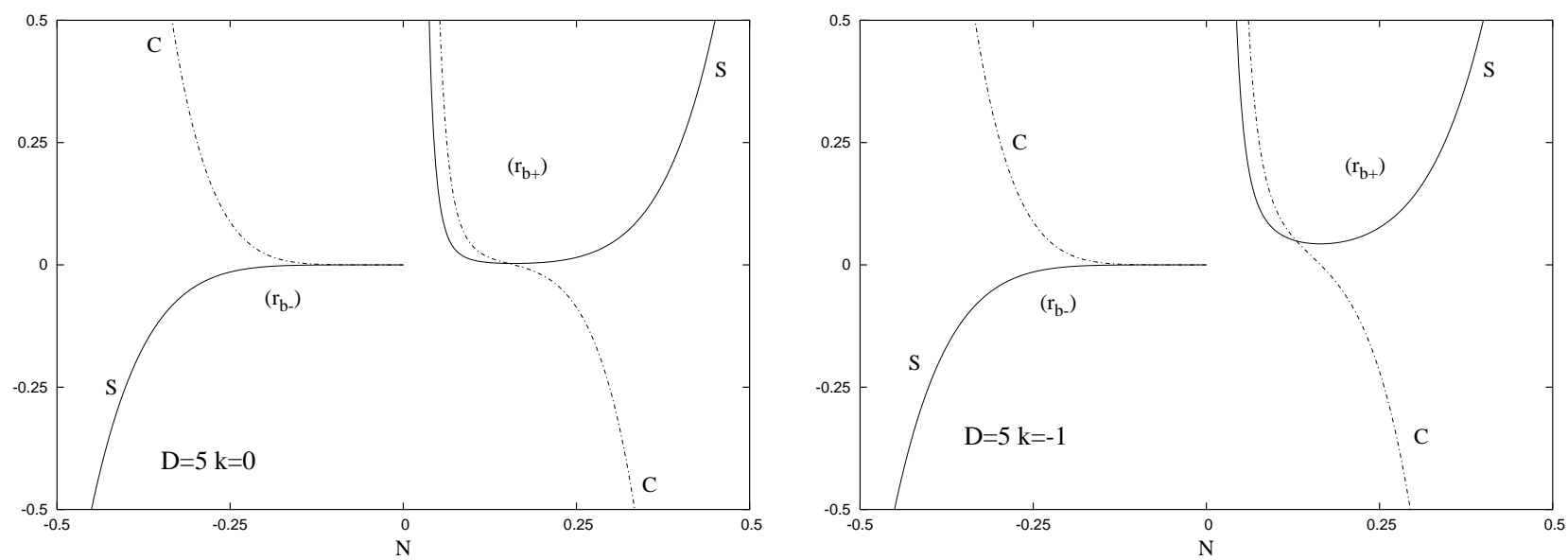

Figure 2. The entropy and specific heat of $k=0,-1$ solutions are represented as a function of $N$ for bolt solutions in six dimensions.

In Figure 2, we ploted the entropy and specific heat per unit volume as function of $N$ for $\ell=1,|\sigma|=1.5$ for topological bolt solutions in six dimensions. In the lower branch case, the relative sign of $(S, C)$ alternates between spacetime dimensionalities of $4 m$ and $(4 m+2)$.

Also, for all studied cases, the entropy is not proportional to the area, despite the absence of Misner strings.

\section{Properties of the boundary metric}

Typically the boundary of this kind of spacetime will be an asymptotic surface at some large radius $r$. However the metric restricted to the boundary $\gamma_{i j}$ diverges due to an infinite conformal factor $r^{2} / \ell^{2}$, and so the metric upon which the dual field theory resides is usually defined using the rescaling

$$
h_{a b}=\lim _{r \rightarrow \infty} \frac{\ell^{2}}{r^{2}} \gamma_{a b}
$$

Corresponding to the boundary metric $h_{a b}$, the stress-energy tensor $\left\langle\tau_{a b}\right\rangle$ for the dual theory can be calculated using the following relation 37]

$$
\sqrt{-h} h^{a b}<\tau_{b c}>=\lim _{r \rightarrow \infty} \sqrt{-\gamma} \gamma^{a b} T_{b c} .
$$

Employing the prescription (4.1) we find the general line element of the dual field theory for a Lorentzian $(D+1)$-dimensional TNAdS solution

$$
d s^{2}=\ell^{2}\left(d \theta_{i}^{2}+f_{k}^{2}\left(\theta_{i}\right) d \varphi_{i}^{2}\right)-\left(4 n f_{k}^{2}\left(\frac{\theta_{i}}{2}\right) d \varphi_{i}+d t\right)^{2},
$$

where again the index $i$ goes from 1 to $p$ and $f_{k}(\theta)$ is given by eq. (2.3). Here it is convenient to use the new parameters $m=1 / \ell, \Omega=n / \ell^{2}$ and to define new "radial" coordinates $\rho_{i}=\ell \theta_{i}$. 
With these conventions, the $D$-dimensional line element (4.3) becomes

$$
d s^{2}=d \rho_{i}^{2}+\frac{f_{k}^{2}\left(m \rho_{i}\right)}{m^{2}} d \varphi_{i}^{2}-\left(\frac{4 \Omega}{m^{2}} f_{k}^{2}\left(\frac{m \rho_{i}}{2}\right) d \varphi_{i}+d t\right)^{2} .
$$

\subsection{The three dimensional case}

We start again with a discussion of the well-known three dimensional case $(p=1)$. For $k=-1$ the corresponding line element is given by

$$
d s^{2}=d \rho^{2}+\frac{\sinh ^{2}(m \rho)}{m^{2}} d \varphi^{2}-\left(\frac{4 \Omega}{m^{2}} \sinh ^{2}\left(\frac{m \rho}{2}\right) d \varphi+d t\right)^{2},
$$

which is the standard form used in the literature of the nontrivial $(2+1)$-dimensional part of a hyperbolic homogeneous Gödel-type universe $\mathcal{G}_{3}$ [11], which can also be viewed as a squashed $\mathrm{AdS}_{3}$ solution [38]. The four-dimensional metric is the direct Riemannian sum $d z^{2}+d s^{2}$ of a flat factor and the $(2+1)$-dimensional metric (4.5). However, the three dimensional metric part contains all the interesting effects exhibited by a four-dimensional Gödel-type spacetime. The famous Gödel rotating universe [12] corresponds to the case $m^{2}=2 \Omega^{2}\left(\ell^{2}=2 n^{2}\right)$. The value $m^{2}=4 \Omega^{2}\left(\ell^{2}=4 n^{2}\right)$ is $\mathrm{AdS}_{3}$ spacetime written in rotating coordinates; the four dimensional generalization is known as the Rebouças-Tiomno space-time [39].

The boundary spacetime of a $k=0$ topologically-Taub-NUT-AdS solution corresponds to the nontrivial $(2+1)$-dimensional part of the Som-Raychaudhuri spacetime 40]

$$
d s^{2}=d \rho^{2}+\rho^{2} d \varphi^{2}-\left(\Omega \rho^{2} d \varphi+d t\right)^{2}
$$

which also has some relevance in a string theory context 41].

According to the terminology in ref. [11], there are also circular $(k=1)$ metrics

$$
d s^{2}=d \rho^{2}+\frac{\sin ^{2}(m \rho)}{m^{2}} d \varphi^{2}-\left(\frac{4 \Omega}{m^{2}} \sin ^{2}\left(\frac{m \rho}{2}\right) d \varphi+d t\right)^{2},
$$

corresponding to the boundary of a $k=1$ four dimensional Taub-NUT-AdS solution. Their properties are extensively discussed in ref. [11]. These metrics can formally be obtained by taking $m \rightarrow i m$ in eq. (4.7), while the line element (4.6) corresponds to a limit $m \rightarrow 0$.

A well known property of the line elements 4.5)-(4.7) is the occurrence of CTCs for a range of the parameters $(m, \Omega)$ [1]. The violation of causality is made possible essentially because the metric coefficient $g_{\varphi \varphi}$ assumes negative values for some range of values of $\rho$, an effect induced by the nondiagonal metric term associated with rotation. This is quite different from creating causal anomalies in flat space by identifying the time coordinate or from the standard AdS causal problems. Furthermore, because these spacetimes are homogeneous, there are CTCs through every event (hence the causality violation is not localized to some small region). Also, a study of geodesics has shown that these spacetimes are geodesically complete (and thus singularity free) 42, 43]. 
For $k=1,0$ causality is violated for every $(m, \Omega)$. In the hyperbolic case $k=-1$, causality violation on the boundary metric (4.5) will appear only for $m^{2}<4 \Omega^{2}$, which separates globally hyperbolic spacetimes from causality violating ones.

For $k=0,-1$, a natural choice for the range of the coordinates covering the entire manifold is $-\infty<t<\infty, 0 \leq \rho<\infty, 0<\varphi \leq 2 \pi$. For $k=1$, it is natural to take $0 \leq \rho \leq \pi m, 0<\varphi \leq 2 \pi$ while, similar to the bulk case, the absence of Dirac string singularities imposes a time periodicity $\beta=8 \pi \Omega / \mathrm{m}^{2}$ (which is just the bulk periodicity).

\subsection{Five dimensional Gödel-type solutions}

Five dimensional line-elements of the form (4.4) have recently been of considerable interest, after the discovery [13] of a five dimensional Gödel-type supersymmetric solutions with very similar properties to $k=0$ solution in three dimensions. The line-element of this solution reads

$$
d s^{2}=d \rho_{1}^{2}+\rho_{1}^{2} d \varphi_{1}^{2}+d \rho_{2}^{2}+\rho_{2}^{2} d \varphi_{2}^{2}-\left(\Omega\left(\rho_{1}^{2} d \varphi_{1}+\rho_{2}^{2} d \varphi_{2}\right)+d t\right)^{2}
$$

corresponding to the case $p=2, k=0$ in eq. (4.4). Here again, although $\partial / \partial t$ is an everywhere timelike Killing vector, the coordinate $t$ fails to be a global time function. As explicitly proven in ref. [14], this geometry is geodesically complete and also contains nontrivial CTCs.

Similar solutions can be written for $k= \pm 1$. However, although possessing the same symmetries, these are generally not solutions of the field equations of the minimal supergravity, and new matter fields should be introduced. In this context, note also the existence of a different construction of the five-dimensional Gödel-type line element, within a different metric ansatz [44].

The hyperbolic case $k=-1$ of $\mathcal{G}_{5}$ is also of special interest; its line element is of the form

$$
\begin{aligned}
d s^{2}= & d \rho_{1}^{2}+\frac{1}{m^{2}} \sinh ^{2}\left(m \rho_{1}\right) d \varphi_{1}^{2}+d \rho_{2}^{2}+\frac{1}{m^{2}} \sinh ^{2}\left(m \rho_{2}\right) d \varphi_{2}^{2} \\
& -\left(\frac{4 \Omega}{m^{2}} \sinh ^{2}\left(\frac{m \rho_{1}}{2}\right) d \varphi_{1}+\frac{4 \Omega}{m^{2}} \sinh ^{2}\left(\frac{m \rho_{2}}{2}\right) d \varphi_{2}+d t\right)^{2} .
\end{aligned}
$$

The solution with $m^{2}=6 \Omega^{2}$ solves the Einstein equations with a negative cosmological constant $\Lambda=-6 \Omega^{2}$. This solution of minimal gauged supergravity admits only one Killing spinor 45]. It is therefore not maximally supersymmetric (it is not $\mathrm{AdS}_{5}$ ) [46. Furthermore for $m^{2}<4 \Omega^{2}$ the Killing vectors $\partial / \partial \varphi_{i}$ become timelike for large enough values of $\rho_{i}$, while $f\left(x^{i}\right)=t$ is a global time coordinate only for $m^{2}>8 \Omega^{2}$.

\subsection{Properties of the general case}

The line element (4.4) provides a natural generalization of the well-known $\mathcal{G}_{3}$ line element, presenting many similar properties. For every set $\left(\rho_{i}, \varphi_{i}\right)$ and value of $k$, it is natural to take a coordinate range very similar to the $\mathcal{G}_{3}$ case. Also, all $k=1,0$ metrics have nontrivial CTCs passing through every spacetime point (though, as for the Gödel universe, these are 
not geodesics). Therefore these manifolds cannot be foliated by everywhere spacelike surfaces and the classical Cauchy problem is ill posed.

Again, the case $k=-1$ is rather special, since for $m^{2}>4 p \Omega^{2}$ the time coordinate $t$ is a global time function and the spacetimes are globally hyperbolic. It can also be proven that $\mathcal{G}_{2 p+1}$ is a homogeneous spacetime for every choice of $k$.

These singularity-free spacetimes have a Ricci scalar $R=2 p\left(\Omega^{2}-m^{2}\right)$. Let us introduce an orthonormal basis with $d s^{2}=\eta_{i j} \vartheta^{i} \vartheta^{j}$ (where $\vartheta^{\rho^{i}}=d \rho^{i}, \vartheta^{\varphi^{i}}=f_{k}\left(\rho^{i}\right) d \varphi^{i}, \vartheta^{2 p+1}=$ $\left.d t+\frac{4 \Omega}{m^{2}} f_{k}^{2}\left(m \rho_{i} / 2\right) d \varphi_{i}\right)$ with $i, j=1, \ldots p$. In this frame, we find the nonvanishing components of the Einstein tensor

$$
E_{\rho_{i}}^{\rho_{i}}=E_{\varphi_{i}}^{\varphi_{i}}=\Omega^{2}(2-p)+k m^{2}(1-p), \quad E_{t}^{t}=p\left(k m^{2}-3 \Omega^{2}\right) .
$$

Thus the energy-momentum tensor for these spacetimes is that of a perfect fluid $T_{a}^{b}=(P+$ є) $u_{a} u^{b}+P \delta_{a}^{b}$, with pressure $P=\Omega^{2}(2-p)-k m^{2}(1-p)$ and energy density $\epsilon=p\left(3 \Omega^{2}-k m^{2}\right)$ while $u^{a}=\delta_{t}^{a}$ is the velocity of a fundamental class of observers. For $k m^{2}=2 \Omega^{2}(p+1)$ this corresponds to a negative cosmological constant $\Lambda=-p(2 p-1) \Omega^{2}$. It would be interesting the find a matter content compatible with these geometries in a field theory context.

The high degree of symmetry of the line element (4.4) simplifies the analysis of some basic physical processes. For example the geodesic equations can easily be solved in the general case. The geodesic equations have the set of independent first-integrals

$$
\begin{aligned}
\dot{t} & =E-4 \Omega \frac{f_{k}^{2}\left(m \rho_{i} / 2\right)}{f_{k}^{2}\left(m \rho_{i}\right)}\left(L_{i}+\frac{4 \Omega E}{m^{2}} f_{k}^{2}\left(m \rho_{i} / 2\right)\right), \\
\dot{\varphi}_{i} & =\frac{m^{2}}{f_{k}^{2}\left(m \rho_{i}\right)}\left(L_{i}+\frac{4 \Omega E}{m^{2}} f_{k}^{2}\left(m \rho_{i} / 2\right)\right),
\end{aligned}
$$

where $E, L_{i}$ are integration constants implied by the existence of the Killing vectors $\partial / \partial t$ and $\partial / \partial \varphi_{i}$. A superposed dot stands for as derivative with respect to the parameter $\tau$, which is an affine parameter along the geodesics; for timelike geodesics, $\tau$ is the proper time. Thus we find a set of $p$ equations on the form

$$
\dot{\rho}_{i}^{2}=\alpha_{i}^{2}-\frac{m^{2}}{f_{k}^{2}\left(m \rho_{i}\right)}\left(L_{i}+\frac{4 \Omega E}{m^{2}} f_{k}^{2}\left(m \rho_{i} / 2\right)\right),
$$

where $\alpha_{i}$ are constants satisfying the constraint $\sum_{i} \alpha_{i}^{2}=E^{2}-\varepsilon(\varepsilon=1$ or 0 for timelike or null geodesics respectively). This type of equation is known from the study of geodesic motion in $\mathcal{G}_{3}$ spacetimes [43]. All the properties derived there apply directly to the general case. For example the geodesic motion is always bounded in the coordinates $\rho_{i}$, except for those free particles in the hyperbolic family of spacetimes which have $\alpha_{i}^{2}-4 \Omega^{2} E^{2} / m^{2}>0$ (this is possible only for values of $m^{2}>4 \Omega^{2}$ ). The equation (4.10) can be solved by taking $x_{i}=f_{k}^{2}\left(m \rho_{i} / 2\right)$, which leads to the solution

$$
\begin{array}{r}
f_{k}^{2}\left(\frac{m \rho_{i}}{2}\right)=\frac{1}{2\left(4 \Omega^{2} E^{2} / m^{2}+k \alpha_{i}^{2}\right)}\left(\alpha_{i}^{2}-2 \Omega E L_{i}-\alpha_{i} \sqrt{\alpha_{i}^{2}-4 \Omega L_{i} E-k m^{2} L_{i}^{2}}\right. \\
\left.\times \cos m \sqrt{k \alpha_{i}^{2}+\frac{4 \Omega^{2} E^{2}}{m^{2}}}\left(\tau-\tau_{0}\right)\right),
\end{array}
$$


which is valid for all $k=1,0$ solutions and the $k=-1$ solutions with $\alpha_{i}^{2}-4 \Omega^{2} E^{2} / m^{2}<0$. The unbounded hyperbolic motion is given by the equation

$$
\begin{aligned}
& \sinh ^{2}\left(\frac{m \rho_{i}}{2}\right)=\frac{1}{2\left(4 \Omega^{2} E^{2} / m^{2}-\alpha_{i}^{2}\right)}\left(\alpha_{i}^{2}-\right. 2 \Omega E L_{i}-\alpha_{i} \sqrt{\alpha_{i}^{2}-4 \Omega L_{i} E+m^{2} L_{i}^{2}} \\
&\left.\times \cosh m \sqrt{\alpha_{i}^{2}-\frac{4 \Omega^{2} E^{2}}{m^{2}}}\left(\tau-\tau_{0}\right)\right) .
\end{aligned}
$$

Thus, it follows that, similar to $\mathcal{G}_{3}$, for $k=-1$ metrics with $m^{2}<4 \Omega^{2}$ each geodesic observer has an optical horizon, i.e. a maximum spatial distance in a $\left(\rho_{i}, \varphi_{i}\right)$ plane beyond which it is not possible to receive light signals.

The wave equation for a scalar, a Dirac or an electromagnetic field can also easily be solved in term of hypergeometric functions for any $(p, k)$.

\section{Discussion}

In this paper we have considered higher dimensional generalizations of the known four dimensional topological nut and bolt metrics with negative cosmological constant. In these solutions, angular spheres with coordinates $(\theta, \varphi)$ are replaced by planes and hyperboloids.

These spacetimes with nut charges present interesting examples of geometries with CTCs. Unlike the $k=1$ case, the CTCs arise even in the $k=0,-1$ cases were no temporal identifications are made. Analysis of the thermodynamics of such solutions is problematic in a Lorentzian context. However it is fairly straightforward to conduct such an analysis on their Euclidean sections. In particular, gravitational entropy (defined via the relation (3.12)) will be present whenever it is not possible to foliate a given spacetime in the Euclidean regime ${ }^{4}$ by a family of surfaces of constant time [26].

If the topology of the $(D+1)$-dimensional Euclidean section is such that there are fixed points in the $\mathrm{U}(1)$ isometry group generated by the (Euclidean) timelike Killing vector $\xi=$ $\partial / \partial \tau$ then it will not be possible to everywhere foliate the spacetime with surface of constant $\tau$. In general conical singularities will appear unless $\tau$ is assigned the requisite periodicity, yielding the usual relationship between area and entropy provided the co-dimension of the fixed-point set is $(D-1)$. Black hole horizons are bolts but not all bolts are horizons. That is not every Euclidean solution with a bolt has an analytic continuation to a Lorentzian section that is a black hole. However in the presence of NUT charge the usual (and presumed universal) relationship between black hole area and entropy is generalized.

Remarkably enough, the AdS/CFT counterterm approach can be employed to compute conserved quantities and gravitational entropy even in these more general cases [7].

Our investigation of the thermodynamics of the topologically TNAdS solutions has yielded a number of unexpected results. The properties of the four dimensional solutions are somewhat generic. For any dimension, the planar nuts represent extremal backgrounds, with zero

\footnotetext{
${ }^{4}$ Here we refer to a Euclidean geometry with non trivial topology. The topology of a Lorentzian spacetime can change with time, only if there is some pathology, such as a singularity or closed time like curves.
} 
temperature. Furthermore, for negative $\Lambda$ there are no hyperbolic nuts. In addition, we found that the first law of thermodynamics removes the ambiguity in the choice of bolt radius $r_{b}$ and fixes its value as a function of $(N, \ell)$, rather similar to the known $k=1$ spherically symmetric case. This in turn yields the result of eq. (3.5) relating $\beta$ and the NUT charge $N$, the key distinction being that for the $k=1$ case the parameter $\sigma$ is an integer, whereas for the $k=0,-1$ cases it is arbitrary.

The entropy derived within the counterterm approach in all cases $k=1,0,-1$ fails to satisfy the usual entropy-area relationship, despite the absence of Misner string singularities in the latter two cases. This is perhaps the most surprising result of this paper: it seems that the entropy/area relation is always violated in the presence of a NUT charge. We also found the rather unusual situation that, although the $k=0,-1$ solutions possess nonzero angular momenta, these charges do not affect the thermodynamics of the system.

It is a well-known fact that a phase transition occurs for hot AdS. That is, at a critical temperature, the thermal radiation in AdS will collapse forming a black hole. Equivalently, the low temperature black holes are not stable and global AdS spacetime is then the preferred state. On the other hand, high temperature large black holes are stable ${ }^{5}$ they do not decay to global AdS spacetime. From the gauge theory viewpoint, this is a confinement/deconfinement phase transition 48 .

The thermodynamics of spherical Taub-Bolt-AdS resembles the Schwarzschild-AdS case. That is, there is a phase transition between $k=1$ Taub-Bolt-AdS and Taub-NUT-AdS. The idea is that one must consider all possible spacetime solutions with the appropriate boundary conditions - possible saddle points in the Euclidean path integral over gravity in the bulk (Taub-Bolt-AdS and Taub-NUT-AdS in our case). The Taub-NUT-AdS metric dominates the path integral at low temperatures, whereas the the Taub-Bolt-AdS metric dominates at high temperatures.

However, unlike the spherical $(k=1)$ Taub-Bolt-AdS spaces, the planar $(k=0)$ TaubBolt-AdS spaces have the property that they do not exhibit a phase transition at finite temperature. ${ }^{6}$

\footnotetext{
${ }^{5}$ This was observed by Hawking and Page 447. Euclidean sections of black hole metrics are periodic in the imaginary time and so, they represent black holes in equilibrium with thermal radiation. However, the thermal radiation in asymptotically flat spaces (all the way to infinity) will have an energy density that will curve the spacetime - make it an expanding or collapsing Friedmann universe. Then, a static situation can be obtained if one considers a black hole in a finite sized box. On the other hand, it is well-known that Schwarzschild black holes have negative specific heat - they absorb faster than they radiate and the equilibrium is unstable. Working in AdS spacetime, one solves both problems: the gravitational potential in AdS space acts like a confining box and black holes larger than the radius of curvature of AdS have positive specific heat (and are presumably stable).

${ }^{6}$ As emphasized in ref. [49], even if classical (Euclidean) $A d S_{5}$ foliations by $R^{4}, S^{4}$ and $H_{4}$ are related by coordinate transformations, the corresponding gauge theories are physically inequivalent — there is a phase transition for $k=1$ but not for $k=0$ (in this case there is a phase transition if one considers the AdS soliton instead of AdS [50]). Different space-like foliations of the background geometry lead to different definitions of time (and the Hamiltonian) of a quantum system. The classical supergravity background (large $N$ limit and large 't Hooft coupling) is equivalent to the full quantum gauge theory on the corresponding slices.
} 
One determines if there is a phase transition by comparing the Euclidean action of the Taub-Nut-AdS and Taub-Bolt-AdS metrics. For $k=0$, we obtain

$$
I_{b}-I_{n}=-\frac{V D \beta}{16 \pi G \ell^{2}} \int_{N}^{r_{b}} d s \frac{\left(s^{2}+N^{2}\right)}{2 s^{2}}\left(s^{2}-N^{2}\right)^{(D-1) / 2},
$$

which is a negative quantity for any choice of $(N, \ell)$.

We also considered the basic properties of the boundary metric of the Taub-NUT-AdS Lorentzian solutions. This led to a natural $\mathcal{G}_{n}$ generalization to higher dimensions of the famous $\mathcal{G}_{3}$ Gödel-type geometry. These spacetimes also contain CTCs for a large range of the parameters, and are interesting especially in connection with the recently discovered five dimensional supersymmetric Gödel solution.

Our computations have been carried out in the Euclidean section. At this point we remark that, on the CFT side, the analytic continuation (3.1) corresponds on the boundary to the Euclidean approach to field quantization in the presence of CTCs, originally proposed by Hawking [51]

$$
t \rightarrow i \tau, \quad \Omega \rightarrow i a
$$

In this approach we analytically continue the Lorentzian spacetime to get a metric with a real Euclidean section. On this section, all the field operators commute and the Green functions are well defined. Explicit values of field operators can be defined by analytic continuation from the Euclidean spacetime. Here we remark that in the presence of off-diagonal terms of the metric tensor, Wick rotation is more problematic and generally involves the analytic continuation of more parameters than the time coordinate (e.g., the angular momentum $J$ for a Kerr-Newmann black hole). For a Gödel-type spacetime, we have to supplement the analytic continuation $t \rightarrow i \tau$ with a continuation in the rotation parameter $\Omega$. However, in contrast to the asymptotic meaning of rotation (as opposed to the angular momentum $J$ ) for a Kerr-Newmann black hole, in this case the rotation has a well defined local character.

By analytic continuation, we obtain a Euclidean metric of the general form

$$
d s^{2}=d \rho_{i}^{2}+\frac{f_{k}^{2}\left(m \rho_{i}\right)}{m^{2}} d \varphi_{i}^{2}+\left(\frac{4 a}{m^{2}} f_{k}^{2}\left(\frac{m \rho_{i}}{2}\right) d \varphi_{i}+d \tau\right)^{2}
$$

which is also (after a suitable rescaling) the boundary of the $(D+1)$-dimensional Euclidean TNAdS spacetime.

It would be desirable to compute some quantities in a Euclideanized Gödel-type background and to compare the results with the bulk predictions, in order to see how much the supergravity "knows" about the boundary CFT. Since in the cases of interest in this paper the dual conformal field theories are poorly known, it is impossible to discuss them in detail. The best one can do at this point is to compare the thermodynamics of a scalar field (toy example) on a Euclideanized Gödel-type background with the results obtained on the gravity side.

Given the high degree of symmetry of the boundary metrics, the well-known formalism of zeta function regularization [52] and its recently proposed generalization (the "local zeta 
function approach" [53]) are especially well suited. This gives rigorous results for the effective action, the vacuum expectation value of the field fluctuations and $\left\langle\tau_{b}^{a}\right\rangle$ for a quantum field propagating in a zero- or finite temperature Euclideanized Gödel-type background. Unfortunately, the computations are quite complicated since the involved series do not occur in the literature. For $k=1$, this procedure implies a generalization of the methods presented in ref. [54] for $D=3$. To our knowledge, the cases $k=0,-1$ have not been discussed in the literature.

A computation of the effective action for a scalar field propagating in a zero-temperature Euclidean $\mathcal{G}_{2 p+1}$ background with $k=0$ (including the five-dimensional supersymmetric case [13]) is presented in Appendix B. A direct application of the relations (B.5), (B.20) leads to an effective action for a massless, minimally coupled scalar field in three and five dimensions

$$
\begin{aligned}
& I_{e f f}^{D=3}=3 \beta V l^{2} a \zeta_{R}^{\prime}(-2) \simeq-0.0913454 \beta V l^{2} a, \\
& I_{e f f}^{D=5}=-\beta V l^{4} a\left(\zeta_{R}^{\prime}(-2)+2 \zeta_{R}^{\prime}(-4)\right) \simeq 0.00482694 \beta V l^{4} a,
\end{aligned}
$$

the general expression of the effective action being on the form

$$
I_{e f f} \sim \beta V \ell^{D-1} a,
$$

where $\zeta_{R}(s)$ is the usual Riemann zeta function. Here $\beta$ is some (large-) periodicity of the coordinate $\tau$ necessary to ensure the orthonormality of the scalar field eigenfunctions. Corresponding expressions for the massive, nonminimally coupled case can also be written by using (B.5, B.24). These results would make the connection with the $(D+1)$-dimensional $k=0$ NUT case, since from the AdS/CFT correspondence we expect $I_{\text {eff }}$ for a massless, conformally coupled scalar field to have a similar form to the bulk action (3.24) (we recall that $a=N / \ell^{2}$. We note here that the bulk actions contain a $(N / \ell)^{D-1}$ scaling factor, as compared to the above $I_{\text {eff }}$. We also found the same signs for the actions.

Although the extrapolation of the zeta function expression (B.24) to the massless, conformally coupled case is not straightforward, since the corresponding scalar field spectrum contains negative eigenvalues, we do not expect to find there a different result. Based on the results in Appendix B, we predict in this case a dependence of the effective action on the parameters $(a, \ell)$ on the form (5.2), and the same different scaling factors $(N / \ell)^{D-1}$. Thus, the overall normalization of the action seems to require a knowledge of physics at string scale curvatures.

The calculations are done in different regimes: the weakly coupled regime for the CFT (open string) and the strongly coupled regime for gravity (closed string). However, the results appear to be remarkably close. An explanation could be the fact that there are no phase transitions as a function of temperature. Then, the theory would appear to be free from drastic changes of degrees of freedom as the coupling is increased.

We close with some comments on the dual Lorentzian conformal field theory. Over the last decade, the issue of causality violation has been extensively discussed in the literature, with special attention being paid to field quantization on a spacetime background that contains CTCs (see ref. [55] for a recent review). It was found that such spacetimes are usually 
unstable with respect to quantum fluctuations. However, most of the papers deal with confined causality violating spacetimes, i.e., the CTCs are confined within some region and there exists at least one region free of them. Regions with CTCs are separated from those without CTCs by Cauchy horizons. Obviously, this is not the case for the Gödel spacetime where causality violation is not a result of the evolution of certain initial data, but rather has existed "forever".

Field quantization in a Gödel-type spacetime has received scant attention in the literature. The difficulties in the standard field quantization in Gödel universe have been explicitly pointed out by Leahy in ref. [56] and consist mainly of the absence of a complete Cauchy surface and of the incompleteness of the mode solutions to the field equations. Despite some attempts, the meaning of a quantum field theory in this background is still unclear.

This issue is interesting especially in connection with the chronology protection conjecture [57]. A repeated number of attempts have suggested that the back reaction of the (divergent) energy momentum tensor of a test field on the spacetime geometry, via the semi-classical Einstein equations, could furnish a possible mechanism for the enforcement of chronology protection. The status of this conjecture in a Gödel-type background is still unsettled.

We argue here that a new perspective on this issue may come from a rather unexpected direction: the conjectured AdS/CFT correspondence. Assuming the validity of this conjecture, the NUT charged AdS solutions provide an opportunity for the study of quantum field theories on Gödel-type rotating spacetimes, which is clearly an interesting subject. Implicitly, a consistent formulation of the field quantization in a Gödel-type background should be possible (although physical intuition tells us that such a theory should present some pathological properties). According to the standard approach, this is possible only for causally well behaved metrics (corresponding for $\mathcal{G}_{3}$ case to $k=-1, m^{2} \geq 4 \Omega^{2}$ ).

Of course, similar to many other situations we do not know here the dual CFT theory. However, we can use the AdS/CFT "dictionary" to predict qualitative features of a quantum field theory in these backgrounds. For example, as implied by this correspondence, the boundary stress tensor in the AdS-NUT space should match the expectation value of the dual CFT. For the four-dimensional Lorentzian line element (2.1), the prescription (4.2) gives for the stress tensor of the dual theory in a $\mathcal{G}_{3}$ background a simple expression

$$
<\tau_{b}^{a}>=\frac{M m^{2}}{8 \pi G}\left[3 u^{a} u_{b}+\delta_{b}^{a}\right]
$$

where $u^{a}=\delta_{t}^{a}$. This tensor is finite, covariantly conserved and manifestly traceless (and is the standard form for the stress tensor of a $(2+1)$-dimensional CFT). Applying the same procedure to a six-dimensional TNAdS metric yields a somewhat similar result for the stress tensor of the dual theory in the five-dimensional supersymmetric $\mathcal{G}_{5}$ background

$$
<\tau_{b}^{a}>=\frac{M m^{4}}{8 \pi G}\left[5 u^{a} u_{b}+\delta_{b}^{a}\right]
$$


which is also finite in every spacetime point, even when the CTCs appear. Thus CTCs do not necessarily mean that the energy-momentum tensor must diverge. ${ }^{7}$ Hence we cannot use this argument as support for the proposal that causality violation occurring in a Gödeltype spacetime might be removed by quantum effects. This situation is different from the confined causality violating spacetimes, where the energy momentum tensor of a quantum field diverges at the Cauchy horizon - unlike Gödel-type spacetimes, there are geodesic CTCs which dominate quantum amplitudes in a semiclassical picture.

The finiteness of the stress tensor of a CFT on Gödel background and of the effective action on the Euclidean/Lorentzian section (or equivalently the entropy) reveals an important fact: in this case, chronology protection cannot be settled at the level of semiclassical quantum gravity (i.e. the gravity background is classical but the matter fields are quantized).

Since string theory is a proposed fundamental theory of quantum gravity, one may expect that stringy corrections to the low energy effective theory resolve causality violations. The low energy effective equations of string theory have as solutions an abundance of such causality violating geometries. Many attempts have been made to understand or to excise the regions with CTCs by holographic screens [14, 15] or by probing the geometry ${ }^{8}$ with appropriate probes [60]. Such a stringy mechanism for the Goedel-like universe was proposed by Drukker et al. in ref. 61] (further refined in the subsequent work of ref. 62]). Using supertubes 63] as probes in a IIA Gödel universe, they show that there will appear instabilities in the CTC region (despite the fact that supertubes preserve the same supersymmetries as the Gödel-like universe itself), concluding that this geometry is not a good string theory background.

\section{Acknowledgements}

The authors would like to thank Alex Buchel, Igor Drozdov, Nadav Drukker, David Mateos, David Page and Cristian Stelea for valuable discussions. DA also thanks Rob Myers for useful conversations. DA gratefully acknowledges the ongoing hospitality of the University of Waterloo's Department of Physics during this research.

DA is supported by a Dow Hickson Fellowship. The work of RBM was supported in part by the Natural Sciences and Engineering Research Council of Canada. The work of ER was supported by Graduiertenkolleg of the Deutsche Forschungsgemeinschaft (DFG): Nichtlineare Differentialgleichungen; Modellierung, Theorie, Numerik, Visualisierung and EnterpriseIreland Basic Science Research Project SC/2003/390 of Enterprise-Ireland.

\section{A. Misner string analysis for general $k$}

We consider here the question of Misner string singularities for compactified Taub-NUT-AdS spacetimes. Althought we set $D=3$, the discussion below is straightforwardly generalized to other values of $D$ - also, it is not dependent upon the specific form of $F(r)$ in eq. (2.2).

\footnotetext{
${ }^{7} \mathrm{~A}$ similar result has recently been obtained [58] for a one-parameter family of supersymmetric solutions of type IIB supergravity that includes $\operatorname{AdS}_{5} \times S^{5}$; it also has causality-violating configurations.

${ }^{8}$ This resembles the enhançon mechanism (see, e.g., refs. [59]).
} 
Following the analysis of Misner [20], we work with the following set of basis vectors

$$
\begin{aligned}
\omega^{0} & =\sqrt{F(r)}\left(d t+4 n f_{k}^{2}\left(\frac{\theta}{2}\right) d \varphi\right), \\
\omega^{1} & =\frac{d r}{\sqrt{F(r)}}, \\
\omega^{2} & =\sqrt{r^{2}+n^{2}} d \theta \\
\omega^{3} & =\sqrt{r^{2}+n^{2}} f_{k}(\theta) d \varphi,
\end{aligned}
$$

in which case the metric (2.1) becomes

$$
d s^{2}=-\left(\omega^{0}\right)^{2}+\left(\omega^{1}\right)^{2}+\left(\omega^{2}\right)^{2}+\left(\omega^{3}\right)^{2} .
$$

The curvature scalars are finite and so there is no curvature singularity. Inverting eqs. A.1. yields

$$
d t=\frac{1}{\sqrt{F}} \omega^{0}-\frac{4 n f_{k}^{2}\left(\frac{\theta}{2}\right)}{\sqrt{r^{2}+n^{2}} f_{k}(\theta)} \omega^{3} .
$$

Thus, we obtain

$$
-(\nabla t)^{2}=\frac{1}{F}-\frac{(4 n)^{2}}{\left(r^{2}+n^{2}\right)}\left[\frac{f_{k}^{2}\left(\frac{\theta}{2}\right)}{f_{k}(\theta)}\right]^{2},
$$

which will become infinite whenever $\frac{f_{k}^{2}\left(\frac{\theta}{2}\right)}{f_{k}(\theta)}$ diverges. Explicitly we have

$$
\frac{f_{k}^{2}\left(\frac{\theta}{2}\right)}{f_{k}(\theta)}= \begin{cases}\frac{1}{4} \tan ^{2}\left(\frac{\theta}{2}\right), & \text { for } k=1 \\ \frac{\theta^{2}}{16}, & \text { for } k=0 \\ \frac{1}{4} \tanh ^{2}\left(\frac{\theta}{2}\right), & \text { for } k=-1\end{cases}
$$

and so one can easy see that only for $k=1$ is there a singularity. If there are identifications in the metric for the other two values of $k$, this will yield $h(\theta, \phi)=$ constant, i.e. $\theta$ will be some function of $\phi$ at the identification boundaries. For example, a torus can be described as a diamond in the plane with opposite sides identified. We identify the boundary $y=1-x$ of the upper right of the diamond with the boundary $y=-1-x$ on the lower left. This is the same as saying that the curve $\theta(\cos \phi+\sin \phi)=1$ is identified with the curve $\theta(\cos \phi+\sin \phi)=-1$ (writing $x=\theta \cos \phi$ and $y=\theta \sin \phi$ ). Nowhere does this identification make $(\nabla t)^{2}$ singular.

One can look at this in a different way by going to rectangular coordinates. We use the relations

$$
r^{2|k|}\left(d \theta^{2}+f_{k}^{2}(\theta) d \varphi^{2}\right)=d x^{2}+d y^{2}+k\left(d z^{2}-d r^{2}\right)
$$

where the coordinate transformation

$$
\begin{aligned}
& x=r^{|k|} f_{k}(\theta) \cos \phi, \\
& y=r^{|k|} f_{k}(\theta) \sin \phi, \\
& z=r^{|k|} \sqrt{1+k f_{k}^{2}(\theta),}
\end{aligned}
$$


has been employed. With this choice of variables the metric becomes

$$
d s^{2}=-\left(\omega^{0}\right)^{2}+\frac{d r^{2}}{F(r)}+\frac{r^{2}+n^{2}}{r^{2|k|}}\left(d x^{2}+d y^{2}+k\left(d z^{2}-d r^{2}\right)\right)
$$

for all three possible values of $k$. Since $x, y, z$ are all differentiable functions, so is $r$. The only possible singularities in the metric for $F(r) \neq 0$ (i.e. for sufficiently large $r$ ) are the singularities in $\omega^{0}$, which has the form

$$
\omega^{0}=\sqrt{F(r)}\left(d t+\frac{2^{|k|} n}{[r(z+r)]^{|k|}}(x d y-y d x)\right)
$$

and is singular at $z=-r$ only for $k=1$ - for $k=0$ there are no singularities. For $k=-1$, we obtain from eq. (A.6) that $z=r \sqrt{1-f_{-1}^{2}(\theta)} \geq r$, and again there are no singularities. The identifications are always in the $(x, y)$ plane for the $k=0,-1$ cases, and so do not render $\omega^{0}$ singular.

Explicitly for each case we have - for the sphere

$$
\begin{aligned}
x & =r \sin \theta \cos \phi \\
y & =r \sin \theta \sin \phi \\
z & =r \cos \theta \\
\omega^{0} & =\sqrt{F(r)}\left(d t+\frac{2 n}{[r(z+r)]}(x d y-y d x)\right), \\
d s^{2} & =-\left(\omega^{0}\right)^{2}+\frac{d r^{2}}{F(r)}+\frac{r^{2}+n^{2}}{r^{2}}\left(d x^{2}+d y^{2}+\left(d z^{2}-d r^{2}\right)\right),
\end{aligned}
$$

for the torus

$$
\begin{aligned}
x & =\theta \cos \phi, \\
y & =\theta \sin \phi, \\
\omega^{0} & =\sqrt{F(r)}(d t+n(x d y-y d x)), \\
d s^{2} & =-\left(\omega^{0}\right)^{2}+\frac{d r^{2}}{F(r)}+\left(r^{2}+n^{2}\right)\left(d x^{2}+d y^{2}\right),
\end{aligned}
$$

and for the hyperboloid

$$
\begin{aligned}
x & =r \sinh \theta \cos \phi \\
y & =r \sinh \theta \sin \phi \\
z & =r \cosh \theta \\
\omega^{0} & =\sqrt{F(r)}\left(d t+\frac{2 n}{[r(z+r)]}(x d y-y d x)\right), \\
d s^{2} & =-\left(\omega^{0}\right)^{2}+\frac{d r^{2}}{F(r)}+\frac{r^{2}+n^{2}}{r^{2}}\left(d x^{2}+d y^{2}-\left(d z^{2}-d r^{2}\right)\right) .
\end{aligned}
$$

Therefore, the analysis above explicitly indicates an absence of Misner-string singularities for $k=0,-1$. 


\section{B. Scalar field in a $k=0$ Euclidean Gödel-type background}

It would be desirable to compute some quantities in an Euclideanized Gödel-type background and to compare the results with the bulk predictions. Although the details of the boundary CFT will depend on the details of the bulk supergravity theory, the generic properties and possible pathologies are expected to be independent of the precise features of the theory. Therefore, similar to other situations we can restrict ourselves to the simple case of a nonminimally coupled scalar field.

For a scalar field with an action

$$
I[\phi]=-\frac{1}{2} \int\left(\nabla^{\mu} \phi \nabla_{\mu} \phi+M^{2} \phi^{2}+\xi R \phi^{2}\right) \sqrt{g(x)} d^{D} x,
$$

(where $M$ is the scalar field mass and $\xi$ determines the coupling with the scalar curvature $R$ of a $D$-dimensional Gödel-type spacetime) the zeta function approach implies the computation of the the eigenfunctions $\Psi_{N}$ and the eigenvalues $\lambda_{N}$ of the differential second-order selfadjoint operator $A=-\nabla^{\mu} \nabla_{\mu}+M^{2}+\xi R$. The eigenvalue equation $A \Psi_{N}=\lambda_{N} \Psi_{N}$ can easily be solved for every value of $k$ in eq. (5.1) by using the ansatz

$$
\Psi_{N}=e^{-i \omega} \prod_{j=1}^{p} g_{k}\left(\rho_{i}\right) e^{i l_{j} \varphi_{j}} .
$$

In this relation, $l_{i}$ are integers and every function $g_{j}$ is a solution of the equation

$$
\frac{1}{f_{k}\left(m \rho_{j}\right)} \frac{d}{d \rho_{j}}\left(f_{k}\left(m \rho_{j}\right) \frac{d g_{j}}{d \rho_{j}}\right)-\frac{m^{2}}{f_{k}^{2}\left(m \rho_{j}\right)}\left(l_{j}+\frac{4 a \omega}{m^{2}} f_{k}^{2}\left(\frac{m \rho_{j}}{2}\right)^{2}\right) g_{j}=\beta_{j} g_{j},
$$

where $\sum_{j} \beta_{j}=M^{2}+\xi R+\omega^{2}-\lambda_{N}$, the functions $g_{j}$ satisfying certain boundary conditions at the limits of the $\rho_{j}$ intervals.

One consider the series with $s \in C$ (the prime on the sum means that any possible null eigenvalues are omitted)

$$
\zeta(s \mid A)=\sum_{N}^{\prime} \lambda_{N}^{-s}
$$

As is well-known, this series converges provided $\operatorname{Re} s>D / 2$. It is possible to continue the above sum into a meromorphic function of $s$ that is regular at $s=0$ [64]. In a path integral approach, the effective action for a scalar field can be formally expressed as the functional determinant of the operator $A$ as

$$
I_{e f f}=-\frac{1}{2} \ln \operatorname{det}\left(A / \mu^{2}\right)
$$

where $\mu$ is an arbitrary renormalization mass scale coming from the path-integral measure. This determinant however is a formally divergent quantity and needs to be regularized. 
In a zeta function renormalization framework, the regularized determinant reads

$$
\ln \operatorname{det}\left(A / \mu^{2}\right)=-\zeta^{\prime}(0 \mid A)-\zeta(0 \mid A) \ln \mu^{2}
$$

We note that since $\zeta(0 \mid A)=0$ in odd dimensions (which is our case), the dependence on the renormalization scale drops out. Relatively simple expressions for the vacuum expectation values of the field fluctuations and energy momentum tensor can be written by using the local zeta function approach [53].

The series (B.4) can be evaluated relatively easy in the zero temperature limit of $k=0$ solutions. For $k=0$ metrics, we can easily solve the equation (B.3) imposing on $g_{j}\left(\rho_{j}\right)$ the boundary conditions of finiteness at the origin and at infinity, which implies the existence of $p$ more quantum numbers $n_{i}$ (see, also, refs. 65, 60] for a study of the Klein-Gordon equation in a $k=0$ background with $p=1,2$ ). This leads to an eigenvalue expression

$$
\lambda_{N}=\omega^{2}+2 a\left(\omega m_{i}+\left|\omega m_{i}\right|+|\omega|\left(2 n_{i}+p\right)\right)+c
$$

where $\left(N=m_{i}, n_{i}, \omega\right)$, the index $i$ runs from 1 to $p$, and we set $c=M^{2}+\xi R$, with the Ricci scalar $R=-2 p a^{2}$. The massless conformally coupled case corresponds to $c=-a^{2}(2 p-1) / 4$. We note the existence of negative eigenvalues over some range of $(\xi, M)$, which indicates that the ground state may be unstable. The functions $g_{0}\left(\rho_{i}\right)$ in the general eigenvalue expression (B.3) are given by

$$
g_{0}\left(\rho_{i}\right)=\rho_{i}^{\left|m_{i}\right|} e^{-\frac{|\omega| a \rho_{i}^{2}}{2}} L_{\left|m_{i}\right|}^{n_{i}}\left(|\omega| a \rho_{i}^{2}\right)
$$

with $L_{|m|}^{n}$ the Laguerre polynomial. Thus the zero-temperature zeta function expression is 9

$$
\zeta(s)=2 \sum_{i=1}^{p} \int_{0}^{\infty} d \omega \sum_{m_{i}=-\infty}^{\infty} \sum_{n=0}^{\infty}\left(\omega^{2}+2 a \omega\left(m_{i}+\left|m_{i}\right|+2 n_{i}+p\right)+c\right)^{-s}
$$

the finite temperature counterpart corresponding to a multidimensional inhomogeneous Epsteintype zeta function with a truncated range [66].

We start by discussing the minimally coupled, massless case $(c=0)$. Here, when evaluating the integral over $\omega$, we consider a cutoff $\epsilon$ at low $\omega$, eliminating the contribution of the $\omega=0$ null eigenvalue. The apparent divergent integral as $\epsilon \rightarrow 0^{+}$can be made harmless as in refs. [52, 53], after one has fixed Re s large finite, executed the integration and performed the analytic continuation of this result at $s=0$. The integral over $\omega$ is evaluated using the relation 67

$$
\int_{u}^{\infty} \frac{x^{\mu-1} d x}{(1+\beta x)^{\nu}}=\frac{u^{\mu-\nu}}{\beta^{\nu}(\nu-\mu)}{ }_{2} F_{1}\left(\nu, \nu-\mu ; \nu-\mu+1 ;-\frac{1}{\beta u}\right), \quad \operatorname{Re} \mu<\operatorname{Re} \nu,
$$

\footnotetext{
${ }^{9}$ Since the volume of the spacetime diverges, we work here with scalar zeta functions per unit volume.
} 
where ${ }_{2} F_{1}(a, b ; c ; z)$ is the hypergeometric series. Applying the quadratic transformation formula 67

$$
{ }_{2} F_{1}(\alpha, \beta ; 2 \beta ; z)=\left(1-\frac{z}{2}\right)^{-\alpha}{ }_{2} F_{1}\left(\frac{\alpha}{2}, \frac{\alpha+1}{2} ; \frac{\beta}{2} ;\left(\frac{z}{2-z}\right)^{2}\right)
$$

we find

$$
\zeta(s)=\frac{(2 a)^{1-2 s}}{s-\frac{1}{2}} I(s) \Xi
$$

where

$$
I(s)=(1+2 \epsilon)_{2}^{1-2 s} F_{1}\left(s-\frac{1}{2}, s ; s+\frac{1}{2} ; \frac{1}{(1+2 \epsilon)^{2}}\right)
$$

is a regular function of $s$, which converges for $\epsilon>0$, and

$$
\Xi=\sum_{i=1}^{p} \sum_{m_{i}=-\infty}^{\infty} \sum_{n=0}^{\infty}\left(\frac{m_{i}+\left|m_{i}\right|}{2}+n_{i}+\frac{p}{2}\right)^{1-2 s} .
$$

A regularized expression for (B.14) is obtained in several steps. This sum can be rewritten as

$$
\Xi=\sum_{j=0}^{p}\left(-\frac{1}{2}\right)^{p-j}\left(\begin{array}{l}
p \\
j
\end{array}\right) \sum_{m_{i}=0}^{\infty} \sum_{n_{i}=0}^{\infty}\left(m_{1}+\ldots+m_{j}+n_{1}+\ldots+n_{p}+\frac{p}{2}\right)^{1-2 s}
$$

(we replaced here $\left.\sum_{m_{i}=-\infty}^{-1}\left(m_{i}+\left|m_{i}\right|+\alpha\right)=\zeta_{R}(0) \alpha\right)$. The sum over $m_{i}, n_{i}$ entering (B.15) can be simplified by using the relation 69

$$
\sum_{n_{1}, \ldots n_{N}=0}^{\infty}\left[c_{1}\left(n_{1}+\ldots+n_{N}\right)+c\right]^{-s}=c_{1}^{-s} \sum_{l=0}^{\infty}(N+l-2 l) \zeta_{H}\left(s, c / c_{1}+p\right),
$$

where $\zeta_{H}(s, a)$ are the Hurwitz zeta functions, which are meromorphic functions with a unique simple pole at $s=1$. However, an evaluation of $\Xi$ may be obtained most easily using its representation in terms of the Barnes zeta function 68

$$
\zeta_{\mathcal{B}}(s, a \mid \vec{r})=\sum_{\vec{m}=0}^{\infty} \frac{1}{(a+\vec{m} \cdot \vec{r})^{s}}
$$

valid for Re s $>$ d, with the $d$-vectors $\vec{m}$ and $\vec{r}$. It is possible to continue the above sum into a meromorphic function of $s$, the only poles of $\zeta_{\mathcal{B}}(s, a \mid \vec{r})$ being for $s=1, \ldots d$, with

$$
\operatorname{Res} \zeta_{\mathcal{B}}(n, a \mid \vec{r})=\frac{(-1)^{d+n} B_{d-n}^{(d)}(a \mid \vec{r})}{(n-1) !(d-n) ! \prod_{j=1}^{d} r_{i}}
$$

while

$$
\zeta_{\mathcal{B}}(-k, a \mid \vec{r})=\frac{(-1)^{d} k ! B_{d+k}^{(d)}(a \mid \vec{r})}{(d+k) ! \prod_{j=1}^{d} r_{i}}
$$


with $p=0,-1, \ldots$, and $B_{a}^{(b)}$ the generalized Bernoulli polynomials.

We find in this way the simple general expression

$$
\begin{aligned}
\zeta^{D=2 p+1}(s) & =\frac{(2 a)^{1-2 s}}{s-\frac{1}{2}} I(s) \sum_{j=0}^{p}\left(-\frac{1}{2}\right)^{p-j}\left(\begin{array}{l}
p \\
j
\end{array}\right) \zeta_{\mathcal{B}}\left(2 s-1, \frac{p}{2} \mid \overrightarrow{1}_{p+j}\right) \\
& =\frac{(2 a)^{1-2 s}}{s-\frac{1}{2}} I(s) \sum_{j=0}^{p}\left(-\frac{1}{2}\right)^{p-j}\left(\begin{array}{c}
p \\
j
\end{array}\right) \sum_{l=0}^{\infty}\left(\begin{array}{c}
p+j+l-2 \\
l
\end{array}\right) \zeta_{H}\left(2 s-1, l+\frac{p}{2}\right) .
\end{aligned}
$$

The above expression can be simplified for particular values of $p$. A useful relation in this context is 69]

$$
\sum_{m, n=0}^{\infty}[m+n+a]^{-s}=\zeta_{H}(s-1, a)-(a-1) \zeta_{H}(s, a) .
$$

The results we find for $p=1,2$ are

$$
\begin{aligned}
& \zeta^{D=3}(s)=\frac{(2 a)^{1-2 s}}{s-\frac{1}{2}} I(s) \zeta_{H}\left(2 s-2, \frac{1}{2}\right)=\frac{(2 a)^{1-2 s}}{s-\frac{1}{2}} I(s)\left(2^{2 s-2}-1\right) \zeta_{R}(2 s-2) \\
& \zeta^{D=5}(s)=\frac{(2 a)^{1-2 s}}{s-\frac{1}{2}} \frac{I(s)}{12}\left(\zeta_{H}(2 s-2,1)+2 \zeta_{H}(2 s-4,1)\right)=\frac{(2 a)^{1-2 s}}{s-\frac{1}{2}} \frac{I(s)}{12}\left(\zeta_{R}(2 s-2)+2 \zeta_{R}(2 s-4)\right)
\end{aligned}
$$

We observe that these series satisfy the standard relation $\zeta(0)=0$ and present simple poles at $s=3 / 2,1 / 2(p=1)$ and $s=5 / 2,3 / 2,1 / 2(p=2)$, in agreement with the general theory. The derivatives of these functions evaluated at $s=0$ are

$$
\zeta^{\prime D=3}(0)=6 a \zeta_{R}^{\prime}(-2), \quad \zeta^{\prime D=5}(0)=-\frac{2 a}{3}\left(\zeta_{R}^{\prime}(-2)+2 \zeta_{R}^{\prime}(-4)\right)
$$

The zeta function for the massive nonminimally coupled case can be constructed following the result (B.20). By using an expansion in terms of the parameter $c$, we find

$$
\begin{aligned}
\zeta_{c}^{D=2 p+1}(s)= & \frac{2(2 a)^{1-2 s}}{\Gamma(s)} \sum_{k=0}^{\infty}(-1)^{k}\left(\frac{c}{a^{2}}\right)^{k} \frac{1}{k !} \frac{\Gamma(s+k)}{2 s+2 k-1} I(s+k) \\
& \times \sum_{j=0}^{p}\left(-\frac{1}{2}\right)^{p-j}\left(\begin{array}{c}
p \\
j
\end{array}\right) \zeta_{\mathcal{B}}\left(2 s+2 k-1, \frac{p}{2} \mid \overrightarrow{1}_{p+j}\right) .
\end{aligned}
$$

These zeta functions are analytic throughout the $s$-complex plane except for $s=D / 2-n$ (with $n=0,1, \ldots$ ) where simple poles appear.

Based on the above results, we can easily compute the effective action for a scalar field with $M^{2}+\xi R \geq 0$. Unfortunately, we cannot use eq. (B.24) in the physically more interesting massless conformally coupled case, since $c<0$ in this limit and both null and negative eigenvalues $\lambda_{N}<0$ are present. However, the zeta function approach can be generalized to the case of negative eigenvalues (see, e.g., refs. [70, 71]). In this case, the sum (B.4) is taken over $\left|\lambda_{N}\right|^{-s}$, a nontrivial phase factor also appearing [71]. 
It can easily be seen that, for the massless conformally coupled case, the $a$ dependence of the zeta function factorizes and it is similar to the massless case, $\zeta(s) \sim a^{1-2 s}$, the sum over $N=\left(n_{i}, m_{i}, \omega\right)$ giving a function of $s$ only, whose evaluation requires a separate discussion. Therefore, we expect the derivative of this function evaluated at $s=0$ (thus the effective action of a massless conformally coupled scalar field) to be proportional to $a$.

\section{References}

[1] J. M. Maldacena, "The large N limit of superconformal field theories and supergravity," Adv. Theor. Math. Phys. 2 (1998) 231, Int. J. Theor. Phys. 38 (1999) 1113 [arXiv:hep-th/9711200]; E. Witten, "Anti-de Sitter space and holography," Adv. Theor. Math. Phys. 2 (1998) 253 [arXiv:hep-th/9802150];

S. S. Gubser, I. R. Klebanov and A. M. Polyakov, "Gauge theory correlators from noncritical string theory," Phys. Lett. B 428 (1998) 105 [arXiv: hep-th/9802109].

[2] O. Aharony, S. S. Gubser, J. M. Maldacena, H. Ooguri and Y. Oz, "Large N field theories, string theory and gravity," Phys. Rept. 323 (2000) 183 [arXiv:hep-th/9905111].

[3] R. Emparan, C. V. Johnson and R. C. Myers, "Surface terms as counterterms in the AdS/CFT correspondence," Phys. Rev. D 60 (1999) 104001 [arXiv:hep-th/9903238].

[4] S.W. Hawking, G. F. R. Ellis, The large structure of space-time, Cambridge, Cambridge University Press, (1973).

[5] A. Chamblin, R. Emparan, C. V. Johnson and R. C. Myers, "Large N phases, gravitational instantons and the nuts and bolts of AdS holography," Phys. Rev. D 59 (1999) 064010 [hep-th/9808177].

[6] R. B. Mann, "Topological black holes: Outside looking in," [arXiv:gr-qc/9709039];

J. P. Lemos, "Rotating toroidal black holes in anti-de Sitter spacetimes and their properties," [arXiv:gr-qc/0011092].

[7] R. Clarkson, L. Fatibene and R. B. Mann, "Thermodynamics of (d+1)-dimensional NUT-charged AdS spacetimes," Nucl. Phys. B 652 (2003) 348 [arXiv:hep-th/0210280].

[8] R. Mann and C. Stelea, "Nuttier (A)dS black holes in higher dimensions," Class. Class. Quant. Grav. 21 (2004) 2937 [arXiv:hep-th/0312285].

[9] E. Radu and D. Astefanesei, "Quantum effects in a rotating space-time," Int. J. Mod. Phys. D 11 (2002) 715 [arXiv: gr-qc/0112029].

[10] E. Radu, "Gravitating non-abelian solutions with NUT charge," Phys. Rev. D 67 (2003) 084030 [arXiv:hep-th/0211120].

[11] M. J. Reboucas and J. Tiomno, "On The Homogeneity Of Riemannian Space-Times Of Godel Type," Phys. Rev. D 28 (1983) 1251.

[12] K. Gödel, "An Example Of A New Type Of Cosmological Solutions Of Einstein's Field Equations Of Graviation," Rev. Mod. Phys. 21 (1949) 447.

[13] J. P. Gauntlett, J. B. Gutowski, C. M. Hull, S. Pakis and H. S. Reall, "All supersymmetric solutions of minimal supergravity in five dimensions," Class. Quant. Grav. 20 (2003) 4587 [arXiv:hep-th/0209114]. 
[14] E. K. Boyda, S. Ganguli, P. Horava and U. Varadarajan, "Holographic protection of chronology in universes of the Goedel type," Phys. Rev. D 67 (2003) 106003 [arXiv:hep-th/0212087].

[15] D. Brecher, P. A. DeBoer, D. C. Page and M. Rozali, "Closed timelike curves and holography in compact plane waves," JHEP 0310 (2003) 031 [arXiv:hep-th/0306190].

[16] E. G. Gimon and A. Hashimoto, "Black holes in Goedel universes and pp-waves," Phys. Rev. Lett. 91 (2003) 021601 [arXiv:hep-th/0304181];

K. Behrndt and D. Klemm, "Black holes in Goedel-type universes with a cosmological constant," [arXiv:hep-th/0401239];

D. Brecher, U. H. Danielsson, J. P. Gregory and M. E. Olsson, "Rotating black holes in a Goedel universe," JHEP 0311 (2003) 033 [hep-th/0309058];

E. G. Gimon, A. Hashimoto, V. E. Hubeny, O. Lunin and M. Rangamani, "Black strings in Asymptotically plane wave geometries," JHEP 0308 (2003) 035 [hep-th/0306131].

[17] M. Novello, N. F. Svaiter and M. E. X. Guimaraes, "Synchronized frames for Godel's universe," Gen. Rel. Grav. 25 (1993) 137.

[18] D. Astefanesei, R. B. Mann and E. Radu, "Breakdown of the entropy/area relationship for NUT-charged spacetimes," [arXiv:hep-th/0406050].

[19] E. T. Newman, L.Tamburino and T. Unti, "Empty space generalization of the Schwarzschild metric," J. Math. Phys. 4 (1963) 915.

[20] C. W. Misner, "Flatter regions of Newman, Unti, and Tamburino's generalized Schwarzschild space," J. Math. Phys. 4 (1963) 924.

[21] C. W. Misner, in Relativity Theory and Astrophysics I: Relativity and Cosmology, edited by J. Ehlers, Lectures in Applied Mathematics, Volume 8 (American Mathematical Society, Providence, RI, 1967), p. 160.

[22] M. Damianski and E. T. Newman, "A combined Kerr-Nut solution of the Einstein field equations, " Bull. Acad. Pol. Sci. 14 (1966) 653;

J. S. Dowker, "The NUT solution as a gravitational dyon," Gen. Rel. Grav. 5 (1974) 603.

[23] R. Emparan, "AdS/CFT duals of topological black holes and the entropy of zero energy states," JHEP 036 (1999) 9906 [arXiv: hep-th/9906040]

[24] F. A. Bais and P. Batenburg, "A New Class Of Higher Dimensional Kaluza-Klein Monopole And Instanton Solutions," Nucl. Phys. B 253 (1985) 162.

[25] A. Awad and A. Chamblin, "A bestiary of higher dimensional Taub-NUT-AdS spacetimes," Class. Quant. Grav. 19 (2002) 2051 [arXiv: hep-th/0012240].

[26] S. W. Hawking, C. J. Hunter and D. N. Page, "Nut charge, anti-de Sitter space and entropy," Phys. Rev. D 59 (1999) 044033 [arXiv:hep-th/9809035];

R. B. Mann, "Misner string entropy," Phys. Rev. D 60 (1999) 104047 [arXiv:hep-th/9903229];

R. B. Mann, "Entropy of rotating Misner string spacetimes," Phys. Rev. D 61 (2000) 084013 [arXiv:hep-th/9904148].

[27] D.A. Konkowski, T.M. Helliwell and L.C. Shepley, "Cosmologies with quasiregular singularities. I. Spacetimes and test waves, " Phys. Rev. D 31 (1985) 1178;

D.A. Konkowski and T.M. Helliwell, "Cosmologies with quasiregular singularities. II. Stability considerations," Phys. Rev. D 31 (1985) 1195. 
[28] S. W. Hawking in General Relativity. An Einstein Centenary Survey, edited by S. W. Hawking and W. Israel, (Cambridge, Cambridge University Press, 1979).

[29] G. W. Gibbons and S. W. Hawking, "Action Integrals And Partition Functions In Quantum Gravity," Phys. Rev. D 15 (1977) 2752.

[30] R. G. Cai and N. Ohta, "Surface counterterms and boundary stress-energy tensors for asymptotically non-anti-de Sitter spaces," Phys. Rev. D 62 (2000) 024006

[arXiv:hep-th/9912013];

V. Balasubramanian, J. de Boer and D. Minic, "Mass, entropy and holography in asymptotically de Sitter spaces," Phys. Rev. D 65 (2002) 123508 [arXiv: hep-th/0110108];

A. M. Ghezelbash and R. B. Mann, "Action, mass and entropy of Schwarzschild-de Sitter black holes and the dS/CFT correspondence," JHEP 0201 (2002) 005 [arXiv: hep-th/0111217];

D. Klemm, "Some aspects of the de Sitter/CFT correspondence," Nucl. Phys. B 625 (2002) 295 [arXiv: hep-th/0106247];

D. Astefanesei, R. Mann, E. Radu, "Reissner-Nordstrom-de Sitter black hole, planar coordinates and dS/CFT," JHEP 0401 (2004) 029 [hep-th/0310273];

E. Radu, Mod. Phys. Lett. A 17 (2002) 2277 [arXiv:gr-qc/0211035].

[31] V. Balasubramanian and P. Kraus, "A stress tensor for anti-de Sitter gravity," Commun. Math. Phys. 208 (1999) 413 [arXiv:hep-th/9902121].

[32] S. Das and R. B. Mann, "Conserved quantities in Kerr-anti-de Sitter spacetimes in various dimensions," JHEP 0008 (2000) 033 [arXiv:hep-th/0008028].

[33] I. S. Booth and R. B. Mann, "Moving observers, non-orthogonal boundaries, and quasilocal energy," Phys. Rev. D 59 (1999) 064021 [arXiv:gr-qc/9810009].

[34] R. B. Mann, "Expanding the Area of Gravitational Entropy," Found. Phys. 33 (2003) 65 [arXiv:gr-qc/0211047].

[35] R. M. Wald, General Relativity, Chicago, Chicago Univ. Press, (1984).

[36] B. Kleihaus, J. Kunz and F. Navarro-Lerida, "Stationary black holes with static and counterrotating horizons," Phys. Rev. D 69 (2004) 081501 [arXiv:gr-qc/0309082].

[37] R. C. Myers, "Stress tensors and Casimir energies in the AdS / CFT correspondence," Phys. Rev. D 60 (1999) 046002 [arXiv: hep-th/9903203].

[38] M. Rooman and P. Spindel, "Goedel metric as a squashed anti-de Sitter geometry," Class. Quant. Grav. 15 (1998) 3241 [arXiv:gr-qc/9804027].

[39] M. J. Reboucas and A. F. Teixeira, "Features Of A Relativistic Space-Time With Seven Isometries," Phys. Rev. D 34 (1986) 2985.

[40] M. M. Som and A. K. Raychaudhuri, "Cylindrically symmetric charged dust distributions in rigid rotation in general relativity," Proc. R. Soc. London A 304 (1968) 81.

[41] G. T. Horowitz and A. A. Tseytlin, "A new class of exact solutions in string theory," Phys. Rev. D 51 (1995) 2896 [arXiv: hep-th/9409021].

[42] S. Chandrasekhar and J. P. Wright "The geodesics in Gödel's universe, " Proc. Nat. Acad. Sci. 47 (1961) 34;

M. Novello, I. D. Soares and J. Tiomno, "Geodesic motion and confinement in Gödel's universe, " Phys. Rev. D 27 (1983) 779. 
[43] M. O. Calvao, I. D. Soares and J. Tiomno, "Geodesics in Gödel-type space-times," Gen. Rel. Grav. 22 (1990) 683.

[44] M. J. Reboucas and A. F. Teixeira, "Causal anomalies in Kaluza-Klein gravity theories," Int. J. Mod. Phys. A 13 (1998) 3181 [arXiv:gr-qc/9808049].

[45] J. P. Gauntlett and J. B. Gutowski, 'All supersymmetric solutions of minimal gauged supergravity in five dimensions," Phys. Rev. D 68 (2003) 105009 [arXiv:hep-th/0304064].

[46] R. Britto-Pacumio, A. Strominger and A. Volovich, "Holography for coset spaces," JHEP 9911 (1999) 013 [arXiv:hep-th/9905211].

[47] S. W. Hawking and D. N. Page, "Thermodynamics Of Black Holes In Anti-De Sitter Space," Commun. Math. Phys. 87 (1983) 577.

[48] E. Witten, "Anti-de Sitter space, thermal phase transition, and confinement in gauge theories," Adv. Theor. Math. Phys. 2 (1998) 505 [arXiv:hep-th/9803131].

[49] A. Buchel and A. A. Tseytlin, "Curved space resolution of singularity of D3-branes on conifold," Phys. Rev. D 65 (2002) 085019 [arXiv: hep-th/0111017].

[50] S. Surya, K. Schleich and D. M. Witt, "Phase transitions for flat adS black holes," Phys. Rev. Lett. 86 (2001) 5231 [arXiv:hep-th/0101134].

[51] S. W. Hawking, "Quantum coherence and closed timelike curves," Phys. Rev. D 52 (1995) 5681 [gr-qc/9502017].

[52] S. W. Hawking, "Zeta Function Regularization Of Path Integrals In Curved Space-Time," Commun. Math. Phys. 55 (1977) 133.

[53] V. Moretti, "Direct zeta-function approach and renormalization of one-loop stress tensors in curved spacetimes," Phys. Rev. D 56 (1997) 7797 [arXiv:hep-th/9705060].

[54] J. S. Dowker, "Effective actions on the squashed three-sphere," Class. Quant. Grav. 16 (1999) 1937 [arXiv:hep-th/9812202].

[55] M. Visser, "The quantum physics of chronology protection," [arXiv:gr-qc/0204022].

[56] D. A. Leahy, "Scalar and neutrino fields in the Gödel Universe," Int. J. Theor. Phys. 21 (1982) 703.

[57] S. W. Hawking, "The Chronology protection conjecture," Phys. Rev. D 46 (1992) 603.

[58] J. P. Gauntlett, J. B. Gutowski and N. V. Suryanarayana, "A deformation of $A d S_{5} \times S^{5}$," arXiv:hep-th/0406188.

[59] C. V. Johnson, A. W. Peet and J. Polchinski, "Gauge theory and the excision of repulson singularities," Phys. Rev. D 61 (2000) 086001 [hep-th/9911161];

D. Astefanesei and R. C. Myers, "A new wrinkle on the enhancon," JHEP 0202 (2002) 043 [hep-th/0112133].

[60] Y. Hikida and S. J. Rey, "Can branes travel beyond CTC horizon in Gödel universe?," Nucl. Phys. B 669 (2003) 57 [arXiv:hep-th/0306148];

D. Israel, "Quantization of heterotic strings in a Gödel/anti de Sitter spacetime and chronology protection," JHEP 0401 (2004) 042 [arXiv:hep-th/0310158];

D. Brace, "Over-rotating supertube backgrounds," [arXiv:hep-th/0310186]; 
L. Dyson, "Chronology protection in string theory," JHEP 0403 (2004) 024

[arXiv:hep-th/0302052];

C. V. Johnson and H. G. Svendsen, "An exact string theory model of closed time-like curves and cosmological singulrities," [arXiv:hep-th/0405141].

[61] N. Drukker, B. Fiol and J. Simon, "Gödel's universe in a supertube shroud," Phys. Rev. Lett. 91 (2003) 231601 [arXiv:hep-th/0306057];

[62] N. Drukker, "Supertube domain walls and elimination of closed time-like curves in string theory," [arXiv:hep-th/0404239].

E. G. Gimon and P. Horava, "Over-rotating black holes, Godel holography and the hypertube," [arXiv:hep-th/0405019].

[63] D. Mateos and P. K. Townsend, "Supertubes," Phys. Rev. Lett. 87 (2001) 011602 [arXiv: hep-th/0103030]

R. Emparan, D. Mateos and P. K. Townsend, "Supergravity Supertubes," JHEP 07 (2001) 011 [arXiv: hep-th/0106012].

[64] E. Elizalde, Ten physical applications of spectral zeta functions (Springer, Berlin, 1995); E. Elizalde, S.D. Odintsov, A. Romeo, A.A. Bytsenko and S. Zerbini, Zeta regularization techniques with applications (World Sci., Singapore, 1994).

[65] B. D. B. Figueiredo, I. D. Soares and J. Tiomno, "Gravitational coupling of Klein-Gordon and Dirac particles to matter vorticity and space-time torsion," Class. Quant. Grav. 9 (1992) 1593.

[66] E. Elizalde, "Multidimensional extension of the generalized Chowla-Selberg formula," Commun. Math. Phys. 198 (1998) 83 [arXiv:hep-th/9707257].

[67] I. S. Gradstein and I. M. Ryshik, Tables of Integrals, Series, and Products, $4^{\text {th }}$ edition (Academic Press, New York, 1965).

[68] E.W.Barnes, "On the theory of multiple gamma function," Trans. Camb. Phil. Soc. 19 (1903) 374.

[69] E. Elizalde, "Multiple Zeta Functions With Arbitrary Exponents," J. Phys. A 22 (1989) 931.

[70] D. V. Vassilevich, "Heat kernel expansion: User's manual," Phys. Rept. 388 (2003) 279 [arXiv:hep-th/0306138];

E. Elizalde, "On two complementary approaches aiming at the definition of the determinant of an elliptic partial differential operator," arXiv:hep-th/9508167.

[71] D. H. Adams and S. Sen, Phys. Lett. B 353 (1995) 495 [arXiv:hep-th/9506079]. 